\title{
Bayesian stroke modeling details sex biases in the white matter substrates of aphasia
}

\author{
Julius M. Kernbach ${ }^{1,2,3}$, MD; Gesa Hartwigsen ${ }^{4}, \mathrm{PhD}$; \\ Jae-Sung Lim ${ }^{5}$, MD; Hee-Joon Bae ${ }^{6}$, MD; Kyung-Ho Yu ${ }^{7}$, MD PhD; \\ Gottfried Schlaug ${ }^{3}$, MD PhD; Anna Bonkhoff ${ }^{8}, \mathrm{MD}$; Natalia S. Rost $^{8}, \mathrm{MD}$; \\ Danilo Bzdok ${ }^{9,10, *}, \mathrm{MD} \mathrm{PhD}$
}

\footnotetext{
${ }^{1}$ Neurosurgical Artificial Intelligence Laboratory Aachen (NAILA), RWTH Aachen University Hospital, Aachen, Germany

${ }^{2}$ Department of Neurosurgery, Faculty of Medicine, RWTH Aachen University, Aachen, Germany

${ }^{3}$ Music, Neuroimaging, and Stroke Recovery Laboratory, Department of Neurology, Beth Israel Deaconess Medical Center and Harvard Medical School, Boston, MA 02215, USA

${ }^{4}$ Max Planck Institute for Human Cognitive and Brain Sciences, Lise Meitner Research Group Cognition and Plasticity, Leipzig, Germany

${ }^{5}$ Department of Neurology, Asan Medical Center, University of Ulsan College of Medicine, Seoul, Republic of Korea

${ }^{6}$ Department of Neurology, Cerebrovascular Center, Seoul National University Bundang Hospital, Seoul National University College of Medicine, Seongnam, Republic of Korea

${ }^{7}$ Department of Neurology, Hallym University Sacred Heart Hospital, Hallym University College of Medicine, Anyang, Republic of Korea

${ }^{8}$ J. Philip Kistler Stroke Research Center, Massachusetts General Hospital, Harvard Medical School, Boston, MA, USA

${ }^{9}$ Department of Biomedical Engineering, McConnell Brain Imaging Centre, Montreal Neurological Institute, Faculty of Medicine, School of Computer Science, McGill University, Montreal, Canada

${ }^{10}$ Mila - Quebec Artificial Intelligence Institute, Montreal, Canada
}

*Corresponding author:

Danilo Bzdok, M.D., Ph.D.

Montreal Neurological Institute

3801 Rue University

McGill University

Montréal (Québec) H3A 2B4

Canada 


\begin{abstract}
Ischemic cerebrovascular events often lead to aphasia. Previous work provided hints that such strokes may affect women and men in distinct ways. Women tend to suffer strokes with more disabling language impairment, even if the lesion size is comparable to men. In 1,401 patients, we isolated data-led representations of anatomical lesion patterns and hand-tailored a Bayesian analytical solution to carefully model the degree of sex divergence in predicting language outcomes $\sim 3$ months after stroke. We located lesion-outcome effects in the left-dominant language network that highlight the ventral pathway as a core lesion focus across different tests of language performance. We provide newly detailed evidence for sex-specific brain-behavior associations in the domain-general networks associated with cortico-subcortical pathways, with unique contributions of the fornix in women and cingular fiber bundles in men. Our collective findings suggest diverging white matter substrates in how stroke causes language deficits in women and men. Clinically acknowledging such sex disparities has the potential to improve personalized treatment for stroke patients worldwide.
\end{abstract}

\title{
Keywords
}

aphasia, ischemic stroke, machine learning algorithms, Bayesian inference, population neuroscience 


\section{Introduction}

Stroke is the leading cause of long-term disability worldwide. By 2047, the prevalence of stroke is estimated to increase by $27 \%$, with women being disproportionately affected (Bushnell et al., 2014; Wafa et al., 2020). Estimations suggest a $~ 55,000$ increase of stroke events in women each year (Bushnell et al., 2014; Giroud et al., 2017). The anticipated rise in stroke prevalence will put a strain on the economic burden of our health systems. The annual stroke-related social and health costs have reached $>60 €$ billion in Europe alone (Luengo-Fernandez et al., 2020; Olesen et al., 2012). Post-stroke aphasia places an overwhelming burden on many of the $>25$ million stroke survivors worldwide (Engelter et al., 2006; Feigin et al., 2017). For affected individuals, the sequelae of stroke can prompt drastic life changes, with severe long-term impairments (Feigin et al., 2014; Pedersen et al., 2004). Aphasia severely impacts everyday communication and social interactions by affecting language production and comprehension. Aphasia is associated with an increased mortality rate (Laska et al., 2001), longer hospitalization, poorer functional recovery, and reduced overall probability of returning to work (Gialanella \& Prometti, 2009).

As an underappreciated fact, women typically suffer strokes with more severe consequences and language impairment even if the lesion size is comparable to men (Hier et al., 1994; Silva et al., 2010). Yet, the majority of stroke lesion studies have been underpowered to detect robust sex disparities given the surfeit of small-sample investigations (Button et al., 2013; Nord et al., 2017). For that reason, placing special emphasis on sex effects requires well-powered patient samples, especially given that many sex-related neural correlates are modest in effect size (Joel et al., 2015). The present investigation in a large patient cohort aims at a direct probabilistic assessment of the subtle sex effects in how the topography of white matter tissue damage leads to differences in language impairments. Such insights on sex-specific lesion-outcome associations are needed to improve personalized treatment prospects in light of the increased burden of aphasia in women.

Aphasia is a frequent consequence of embolic occlusion of the middle cerebral artery. However, brain lesion distributions are highly variable in the preserved and impaired language capacity. In attempting to harmonize the heterogeneous clinical manifestation of aphasia, decades of research efforts have been devoted to determining the complex neural basis of language processing. Today, there is growing consensus that the human language faculty is supported by distributed large-scale networks. Numerous studies have shed light on the anatomical white matter connections at play. In particular, the arcuate fasciculus (AF) has been at the center of attention for decades (Catani \& Mesulam, 2008; Dick \& Tremblay, 2012). Early anatomical dissection outlined its anatomical trajectory, which suggested a direct fiber connection of Wernicke's area in the left temporal lobe to Broca's area in the left frontal lobe (Dejerine et al., 1895; Geschwind, 1970). Applications of diffusion tensor imaging techniques promoted the idea of a further division of the AF into three segments. One 
direct temporo-frontal segment and two lateral segments have been proposed to compose the overall AF. The lateral tract subsegments implement an indirect fronto-parietal and temporal-parietal pathway (anterior and posterior segment, respectively) (Catani et al., 2005; Catani \& Thiebaut de Schotten, 2008). The AF may provide an anchor for the canonical perisylvian network and has been placed at the core of the neuroarchitecture of the uniquely human language abilities (Rilling et al., 2008).

Attempting to marry its structural and functional bases, researchers have integrated the core perisylvian pathway into different functional-anatomical modules. One popular idea is the dual-stream concept of language (G. Hickok \& Poeppel, 2000; Gregory Hickok, 2009; Gregory Hickok \& Poeppel, 2004, 2007; Rauschecker \& Tian, 2000; Saur et al., 2008). This view puts forward a ventral stream for mapping sound-to-meaning and a dorsal stream comprising the AF and superior longitudinal fascicle for mapping sound-to-motor representations. The ventral pathway includes the uncinate fasciculus (UF), the inferior fronto-occipital fasciculus (IFOF), and inferior longitudinal fasciculus (ILF) that together assist in processing speech signals for language comprehension (Catani et al., 2003; Catani \& Mesulam, 2008; Papagno et al., 2011; Saur et al., 2008). The dorsal stream is thought to be left-dominant, while the ventral stream is believed to be more bilaterally organized (Catani \& Mesulam, 2008; Eichert et al., 2019; Gregory Hickok \& Poeppel, 2007). However, there is strong interindividual variability in the degree of hemispheric dominance in general, and accumulating evidence points towards aspects of sex-specific differentiation in particular. For example, more pronounced left lateralization in the long segment of the AF was found in men (Thiebaut de Schotten et al., 2011). Sex differences have also been reported for lateralization of functional activation during carefully designed language tasks (Shaywitz et al., 1995).

Clinically, ischemic stroke generally affects male and female patients differently in terms of incidence, severity, survival, and potential for recovery (Bonkhoff, Karch, Weber, Wellmann, \& Berger, 2021; Bushnell et al., 2014; Dehlendorff et al., 2015). In fact, women generally suffer strokes with more severe neuropsychological consequences compared to those of men (Bonkhoff, Schirmer, Bretzner, Hong, et al., 2021; Silva et al., 2010). Similar lesion volume effects were noted in cases of aphasia, in which smaller ischemic stroke lesions were capable of producing aphasia in women than men (Hier et al., 1994). The reasons behind sex idiosyncrasies in functional outcomes after stroke remain elusive. Differences are often explained by poorer pre-stroke function, more comorbidities such as depression, and social isolation at an older age (see (Reeves et al., 2008)). Yet, adjustment for these factors does not adequately explain the observed discrepancies in stroke outcomes between men and women (Di Carlo et al., 2003; Holroyd-Leduc et al., 2000).

Despite the apparent differences in stroke outcome between women and men, sex is often treated as a nuisance variable in the overwhelming majority of stroke lesion studies (Baldo et al., 2013; Mirman et al., 2015; Wu et al., 2015). This established practice blinds researchers to detect any 
potential neuroanatomical effects that depend on sex. Compounding these challenges that hold barriers to understanding sex-specific brain lesion effects, lesion-symptom mapping studies often constrained the analyses to the predominantly affected hemisphere. That is, as a general trend, the left hemisphere is specifically investigated in language impairment and the right hemisphere in neglect (Baldo et al., 2013; Bates et al., 2003; Bonilha et al., 2017; Smith et al., 2013; Wu et al., 2015). To overcome several existing roadblocks, the present work takes advantage of Bayesian principles as a formal device to quantify degrees of sex divergences. This clean methodology enabled sex-aware analysis in 1,401 ischemic stroke patients, covering major white matter pathways in both hemispheres. Understanding how lesion location, structural language lateralization, and their clinical outcome diverge in aphasia between female and male populations can finess personalized rehabilitation and treatment. 


\section{Results}

We go new ways to chart sex disparities in one of the largest existing stroke cohorts with rigorous neuropsychological profiling. We espoused probabilistic Bayesian principles for direct uncertainty quantification of nuanced sex effects on language performance attributable to specific white matter lesions. First, we extracted a low-dimensional embedding of the voxel-granularity MRI-lesion distributions using unsupervised pattern discovery. Second, the patient-specific expressions of the derived lesion configurations provided the basis for a Bayesian model to predict stroke outcomes with its full probabilistic differentiation between women and men.

\section{Patient cohort: characteristics and behavioral outcome measures}

Our inclusion criteria were met by 1,401 patients with ischemic stroke who were recruited as a prospective multi-center cohort from 2007-2018 (Table 1 for details). The mean age was 67.58 (standard deviation 11.59), with $42 \%$ women. The mean age at admission was significantly higher in females than males ( $\mathrm{t}=8.9, \mathrm{p}<0.001$, cf. Table 1). Patients had an average of 9.28 (5.92) years of education, with women amounting to fewer years on average compared to those of men $(\mathrm{t}=-17.27$, $\mathrm{p}<0.001$ ). Based on the IQCODE score, females' cognitive performance before the ischemic event was significantly worse compared to males $(\mathrm{t}=3.91, \mathrm{p}<0.001)$. Stroke severity as reflected in the clinical impairment was significantly higher in females across nine cognitive tests (K-MMSE, BN, SF, PF, RCFT, RCFT delayed recall, digital symbol coding, TMT-A, and -B). Out of 15 objects to be recognized in the BN (Kaplan et al., 1983), patients correctly named 9.92 (3.59) on average, with females scoring worse than males $(\mathrm{t}=-11.90, \mathrm{p}<0.001)$. Females also performed worse in semantic and phonemic fluency $(\mathrm{t}=-7.12$ and -7.97 , both $\mathrm{p}<0.001)$. In sum, we observed more severe stroke-related deficits and worse premorbid cognitive performance in female patients of our cohort.

\section{Topography of ischemic stroke lesion patterns}

Excellent whole-brain coverage was attested by the voxel-wise summary of lesion overlap across 1,401 patients (Figure 1A). The lesion overlap predominantly corresponded to the vascular territory of the left and right middle cerebral artery. The majority of lesions were located in the deep central white matter in both hemispheres. Most tissue lesions were concentrated in the thalamus and basal ganglia. The average lesion volume was $17.08 \mathrm{ml}(14.81$ - 19.36) and did not significantly differ between female and male patients within our cohort (women: $15.05 \mathrm{ml}$ (11.47 - 18.63), men: $18.57 \mathrm{ml}$ (15.63 - 21.51), $p=0.13$; Table 1). We did not find significant differences between females and males regarding their stroke topography (Figure 1B-C, summarized separately). 


\section{Anatomy of the extracted stroke lesion atoms}

We first extracted hidden topographical archetypes from the high-dimensional lesion distribution in white matter at voxel resolution. Using non-negative matrix factorization (NNMF; (D. D. Lee \& Seung, 1999)) for unsupervised pattern discovery, we uncovered recurring spatial configurations of concurrent tissue damage that are consistently apparent across $>1,000$ stroke cases which we call lesion atoms (Figure 2). Disentanglement of this sum-of-parts representation by means of machine learning provides an important advantage for the purpose of the present study. The non-negativity constraint of the applied algorithm enabled neurobiological meaningful and direct interpretable lesion patterns. In contrast, alternative dimensionality reduction tools such as principal component analysis would hurt an intuitive interpretation of the lesion atom effects. This is because alternative tools recover patterns through incomprehensible positive and negative cancellations of the separate lesion configurations. As a descriptive post-hoc analysis, each lesion atom was assessed for hemispheric dominance of the implicated tissue lesion distribution by an index of lateralization (Figure 2C). For most factors, there was a unique left-hemispheric lesion constellation in correspondence with a similar right-hemispheric homolog (Figure 2A box, factors 3 and 9, or 2 and 5). Overall, the spatial distribution of the derived lesion atoms emerged to be directly interpretable and biologically plausible with components underlying distinct territories of arterial supply (Figure 2).

Four lesion atoms of stroke-induced tissue damage were mapped explicitly to anatomical tracts implicated in the dorsal and ventral language pathways (Figure 2A). Generally, the left-lateralized language atoms showed stronger lesion effects than their right-lateralized homologs. Factor 9 primarily covered white-matter lesions of the core perisylvian pathway with a focus on the left hemisphere. These lesion effects predominantly rested on the AF. The level of relevance within the subsegments of the AF followed an anterior-to-posterior gradient. Here, the anterior and long segments showed higher lesion importance than the posterior segment. The core perisylvian right-hemispheric homolog was captured by factor 3. The organization of the AF's different segments again showed an anterior-to-posterior transition. Both factors implicated the UF pathway to a lesser extent. Factor 5 resembled the left-lateralized ventral pathways, which included the ILF, the IFOF, and the UF. These lesion atom effects further implicated damage in the left optic radiation (OR), and to a lesser degree, the posterior segment of the AF. The right-hemispheric homolog to factor 5 was identified as factor 2. Right-hemispheric (Factor 2) ventral pathways were less pronounced regarding their overall lesion importance compared to the left.

Three lesion atoms resembled damage to the brain's motor circuits (Figure 2A bottom row). The pattern topology of lesion factor 1 included the left-lateralized motor projections of the 
cortico-spinal tract (CST), internal capsule (IC), and cortico-pontine-cerebellar tract (CPC). Similarly, factor 7 captured lesions in the mirrored motor projections of the right hemisphere. Factor 10 was the only factor with no hemispheric dominance and captured bilateral cerebellar connections, including the $\mathrm{CPC}$, the inferior and superior cerebellar peduncle (ICP, SCP). Three further lesion atoms captured the prominent influence of the limbic system. The right cingular fibers dominated the tissue damage configuration of factor 4 , next to a lesser influence of the posterior segment of the AF, IFOF, and IC. Conversely, the lesion constellation of factor 6 captured the left-hemispheric cingular influence. Lastly, factor 8 was dominantly shaped by the influence of the left fornix. Notably, only factor 8 did not have a homologous pattern on the right hemisphere.

By bringing to bear an algorithmic approach from machine learning, we deconvolved unique lesion atoms as low-dimensional representations of stroke configurations from 1,401 patients. Out of ten lesion atoms, four atoms specifically resembled the ventral and dorsal core language pathways on the left and homologous right hemisphere. Three resembled motor pathways, and three further lesion atoms predominantly coincided with limbic pathways in the fornix and cingular fiber pathways.

\section{Lesion atoms track interindividual differences in cognitive outcomes}

To chart the clinical relevance of each lesion atom, we computed Pearson's correlations between patient-specific expressions of a given lesion atom and a cognitive test, one for each available neuropsychological assessment (Figure 3). Based on the ensuing (absolute) factor-outcome association strengths (Figure 3A), we could rank the lesion atoms across all outcome measures of our cohort with unusual deep phenotyping. As a consequence of this bottom-up approach, factors 5 (Ventral pathway R), 8 (Fornix L), and 9 (Dorsal pathway L) ranked as the three most clinically relevant lesion atoms given our functional domains of cognitive outcomes. The limbic factors 4 and 6 reflected the right and left influence of the cingular fiber bundle and ranked fourth and fifth. Except for the TMT, factors 5, 8 , and 9 showed pronounced negative associations across most cognitive scores (Figure 3B). Regarding the language outcomes, damage in the left-hemispheric lesion atoms of the ventral and dorsal pathways (Factors 5 and 9, respectively) caused a significant degree of deterioration in Boston naming (BN), semantic fluency (SF), and phonemic fluency (PF). However, the association with their respective right-hemispheric homologs diverged: Notably, the right hemispheric homolog of the core perisylvian network (Factor 3) showed a slightly negative association with $\mathrm{BN}$, but otherwise positive associations for SF and PF. In contrast, the right-hemispheric homolog of the ventral pathway (Factor 2) was negatively associated with BN and SF, with close links to deteriorated naming and speech fluency. 
We thus ranked the uncovered lesion atoms based on their clinical sequelae. Specifically for language outcomes, we observed diverging responses in the left and right homologs of the ventral and dorsal pathways: for the dorsal perisylvian pathway, severe deterioration was found for left-hemispheric damage (Factor 9) and less for right-hemispheric damage (Factor 3). In contrast, both damage to the left (Factor 5) and right (Factor 2) ventral pathways especially inflicted impairment in both naming and speech fluency.

\section{Sex divergences in lesion atom expressions and prediction of language outcome}

Next, we turned to the direct quantification of degrees of sex differentiation in how the emerged lesion patterns predict language outcome. Based on the patient-specific constellations of lesion pattern expressions, we estimated a fully probabilistic, generative model to explain language outcome at $\sim 3$ months after stroke. In particular, our approach was able to obtain full Bayesian posteriors over the differentiation between females and males. In the present analysis, we focused on the quantitative analyses of the language outcomes of naming ability (BN) and speech fluency (SF and PF; other scores c.f. Supplement Fig. 1-12). Leveraging the Bayesian modeling framework, we provided an honest quantification of a) the general effects of the derived lesion atoms on cognitive outcome after stroke and b) the degree of sex divergence in how the lesion atoms achieve the prediction of post-stroke language outcome. All analyses were adjusted for variation that could be explained by age, age ${ }^{2}$, sex, education in years, pre-morbid cognitive performance, and total lesion volume. We also included sex as a covariate in the model to explicitly account for a-priori differences between female and male stroke outcomes (Bonkhoff, Schirmer, Bretzner, Hong, et al., 2021). For example, worse language outcomes in females could be linked to an underlying cause independent of the lesion distribution, such as a decreased likelihood of receiving acute interventions. In this scenario, the effect would be expressed in the Bayesian posterior distribution of the sex covariate, but not in that of the female-stratified lesion atom.

Among our target language capacities, we first examined interindividual differences in naming ability as captured by the $\mathrm{BN}$ test. The posterior predictive checks confirmed an appropriately fitted approximation of the data variation that underlies how lesion atom expressions explain cognitive performance outcomes $\left(\mathrm{R}^{2}=0.51\right.$, Figure $\left.4 \mathrm{~A}\right)$. We then inspected the role of each lesion atom in forecasting future naming performance in $\mathrm{BN}$ at $\sim 3$ months after stroke, separately for women and men (Figure 5A). Both sexes displayed worse naming outcomes with increasing damage to the left-lateralized ventral pathway (Factor 5), its right-hemispheric homolog (Factor 2), and the right cingular fiber pathway (Factor 4). However, the inferred posterior parameter distributions also attested specific divergences between females and males regarding lesion-outcome associations: the 
right-hemispheric homolog of the core perisylvian network (Factor 3) and the left fornix (Factor 8). Here, damage in the lesion pattern configurations apparent in men was predictive of worse language outcomes. In contrast, stronger impairment in females was further explained by damage in the left core perisylvian network (Factor 9). Tissue damage in the motor projections of the right hemisphere (Factor 7) and the left cingular fiber pathway (Factor 6) explained more pronounced deficits in naming performance in women than men. We thus identified common and diverging effects across the subject expressions of lesion atoms to predict naming capacity between women and men.

Out of all extracted lesion atoms, distinct factors carried explanatory weight jointly for both sexes or uniquely for the female and male patients in our stroke population. The explanatory relevance for $\mathrm{BN}$ was indicated by the inferred marginal posterior parameter distributions (Figure 6A). Most relevant across both sexes, we identified a driving effect in the left-lateralized ventral pathway (Factor 5). Stroke-induced consequences were stronger in women (mean of the posterior distribution $[\mathrm{PM}]=$ -0.251 , highest posterior density interval [HPDI] of the posterior distribution covering $80 \%$ certainty $=-0.410$ to -0.090$)$ than in men $(\mathrm{PM}=-0.158$, HPDI -0.267 to -0.040$)$. We found further sex-diverging lesion-outcome effects. In women, tissue damage in the left fornix (Factor 8) predicted a more preserved outcome (Factor 8 ; PM $=0.138$, HPDI 0.008 to 0.248 ). In males, we identified small but consistent effects: the right homolog of the ventral pathway contributed to the prediction of lost function (Factor 2; PM $=-0.032$, HPDI -0.063 to -0.009 ). Further, damage to the bilateral cerebellar network (Factor 10; PM $=-0.080$, HPDI -0.119 to -0.037 ) contributed to explaining naming impairments. Together, our BN findings witness a dominant lesion-outcome effect in the left ventral language pathway, consistently for both women and men. For women, the left fornix lesion was indicative of more moderate impairment of cognitive capacity $\sim 3$ months after stroke. In contrast, males only showed modest diverging lesion pattern effects located in the homologous right ventral pathway and bilateral cerebellar connections.

Examining how SF outcome differences are underpinned by white matter tissue damage, the posterior predictive check indicated an explained variance of $\mathrm{R}^{2}=0.37$ (Figure 4B). Considering the male versus female lesion-outcome associations, we identified strong effects for both sexes in the left ventral pathway (Factor 5; Figure 5B), followed by the left cingular fibers (Factor 6) and the right homolog of the ventral pathway (Factor 2). Damage captured by each of these three lesion atoms played contributing roles in lost SF function. Lesions affecting the right dorsal perisylvian circuit (Factor 3) explained a more moderate deficit for both sexes. Yet, in the SF function, too, we detected pronounced sex divergences. Men deteriorated more in SF tests as a result of tissue damage in the right cingular fibers (Factor 4). In contrast, white matter damage in the left core perisylvian network (Factor 9) added to difficulties with language fluency in women. 
The lesion patterns predictive of SF differences resembled those explaining BN differences as evidenced by the corresponding marginal posterior distributions: our model attributed SF dysfunction in part to tissue damage in the left ventral pathway (Factor 5, Figure 6B) in both sexes. This lesion-outcome association was more pronounced in women (PM $=-0.360$, HPDI -0.561 to -0.186 ) than men (PM $=-0.190$, HPDI -0.345 to -0.069 ). Patient expressions of the lesion atom in the right-hemispheric perisylvian network were informative for predicting preserved function in women (PM $=0.157$, HPDI 0.034 to 0.276 ) but not in men (Figure 6 left). Similar to the BN analysis, the right ventral pathway (Factor 2, PM $=-0.040$, HPDI -0.069 to -0.010 ) helped predict loss in SF function in males (Figure 6 right). The left-hemispheric motor projections damage (Factor 1, PM = -0.045 , HPDI -0.088 to -0.012 ) helped predict SF dysfunction. However, we observed an even stronger lesion-outcome contribution by the right cingular fibers (Factor 4, PM $=-0.268$, HPDI -0.400 to -0.121) in men. Concluding our SF findings, we found a common lesion-outcome effect in the left ventral pathway. In women, tissue damage compromising the right perisylvian pathway added to the predictability of SF performance outcomes in our patients. Damage caused to the right cingulum assisted in the prediction of future SF loss in men beyond the damage caused to the left ventral pathway.

As the last examined facet of language capacity, we quantified how patterns of tissue damage underpin interindividual differences in PF performance $\sim 3$ months after stroke. The full probabilistic model explained $\mathrm{R}^{2}=0.37$ of the variance in the outcome predictions based on the patient expressions of dedicated lesion atoms (Figure 4B). Common to both sexes, our model attributed lesion damage in the left core perisylvian circuit (Factor 9) to loss of PF function. We found marked divergences between sexes in factors 3-6 (Figure 5). Specifically, language dysfunction in females was due, to a larger extent, to damage to the left ventral pathway (Factor 5). In contrast, men showed more pronounced consequences for cognitive performance due to damage to the right and left cingulum (Factor 5 and 6) and the right perisylvian pathway (Factor 3).

Based on the inferred Bayesian posteriors (Figure 6C), the left dorsal perisylvian pathway showed considerable relevance in explaining differences in PF function, which was comparable in both sexes (Factor 9, females: PM $=-0.151$, HPDI -0.298 to -0.020 ; males: PM $=-0.146$, HPDI -0.273 to -0.029). However, women's functional loss in PF was more explained by damage in the left ventral pathway (Factor 5, PM $=-0.235$, HPDI -0.437 to -0.043 ) compared to men. Additionally, lesions in the bilateral cerebellar network (Factor 10; PM $=-0.058$, HPDI -0.114 to -0.008 ) tracked performance decline in PF. Further, men showed a strong negative effect in the right cingular pathway (Factor 4, PM $=-0.217$, HPDI -0.359 to -0.077). Together, our lesion-outcome effects in PF highlighted the influence of the left-dominant perisylvian network common to both sexes. In women, the left ventral 
pathway added predictive value as to a loss of function in SF. In men, however, the influence of the right cingulum was further indicative of deterioration in $\mathrm{PF}$.

Our sex-sensitive approach enabled us to tease apart shared and diverging lesion-outcome effects in female and male stroke populations. Across both sexes, the ventral pathway was critically involved in naming and semantic fluency deficits, while the dorsal pathway was implicated in phonemic fluency deficits. More generally, we provide novel evidence for sex-nuanced lesion-outcome effects. Concluding our findings across all language performance scores, women tend to rely on left-dominant circuits, including structural connections of the fornix. We further detailed a female-specific vulnerability to ventral lesion damage in phonemic fluency. In contrast, this facet of language performance in men tended to draw from bilateral ventral and cerebellar connections. Damage caused to the right cingular fiber connections assisted in the prediction of future SF loss in men beyond the damage caused to language-specific pathways. 


\section{Discussion}

Language capacity is uniquely evolved in humans and acts as a pacemaker in our societies. There is now an increasing realization that brain tissue damage caused by stroke entails partly distinct consequences on language performance in women and men. We hence designed a fully probabilistic framework as a clean analytical solution to modeling the apparent degrees of sex disparity. First, we extracted neurobiologically meaningful lesion patterns from 1,401 stroke patients in a data-led fashion. Second, we custom-tailored a bespoke Bayesian strategy to partition interindividual variation in caused aphasia outcomes into their lesion pattern contributions. In so doing, we offer honest uncertainty quantification of sex-biased effects in clinical language outcomes after stroke in one of the largest stroke cohorts with rarely available deep phenotyping.

In particular, (1) we identified lesion-outcome effects in men and women in the left-dominant language network, highlighting ventral fiber tracts of the left ILF, the IFOF, and the UF as core lesion focus across loss of different language facets. (2) Across both sexes, our analyses of brain lesion topographies pointed to an overarching structural organization reflecting the dual-stream concept of language and revealed pronounced sex divergencies in the role of lesion patterns. (3) We provide novel evidence for sex-nuanced lesion-outcome effects in the domain-general supra-modal networks, which our model attributed to cortico-subcortical pathways, with strong implications of the fornix and cingular fiber bundles. In the following, we discuss our findings for the anatomical topology of the identified lesion atoms, the shared lesion-outcome effects across sexes, and diverging predictive effects between men and women in implicated domain-general supra-modal networks.

Our analytical approach offered several advantages over classical lesion-symptom mapping studies. In conventional lesion-symptom mapping, each brain voxel is usually considered in isolation (Bates et al., 2003). However, analyzing the contribution of each single brain voxel individually deters intuitive interpretation by omitting interaction effects between spatially distant locations. Multivariate extensions have started to address the problem of mutual dependence between voxels (Pustina et al., 2018; Smith et al., 2013; Sperber, 2020). However, these approaches remain ill-suited to detecting overarching sources of variation that are driving disparate brain effects in continuous degrees, such as brain lateralization or sex-specific lesion-behavior associations. By means of our purpose-designed generative model, we could simultaneously appreciate local tract-lesion effects (and inter-dependencies between them) and their spatially distributed configurations. Leveraging the hierarchy of our fully probabilistic approach, we can directly integrate their hemispheric and sex-specific idiosyncrasies into one unified model for clinical outcome prediction. 
The estimation of our framework was supported by empirical lesion data from a large sample of 1,401 stroke patients with rarely available whole-brain coverage. In line with the existing literature, the lesions were predominantly localized in subcortical zones and led to a critical disconnection of deep white matter tracts (Corbetta et al., 2015). Our data source thus presented two thrusts forward in stroke neuroimaging modeling. First, the spatial topography of the discovered lesion constellations covered both hemispheres. In many previous stroke studies on aphasia or spatial neglect, researchers have limited the anatomical space of investigation to either the left or right hemisphere alone (Baldo et al., 2013; Basilakos et al., 2015; Bates et al., 2003; Harvey \& Schnur, 2015; Mirman et al., 2015; Smith et al., 2013). This unique data scenario enabled us to detect delicate predictive effects, including subtle sex differences, with regard to both brain hemispheres. Second, our findings provide comparable lesion coverage for both sexes. In contrast to the large majority of stroke lesion studies (Baldo et al., 2013; Mirman et al., 2015; Wu et al., 2015), we explicitly model sex as a higher model effect of interest. Potential differences independent of the sex-specific brain damage-behavior relation are further accommodated by our covariates at the lower level of our Bayesian model. This strategy ensures unique lesion-symptom effects for women and men and potentially helps to explain previous seemingly contradictory findings (Cahill, 2006).

Our pattern discovery framework enabled us to identify topologically coherent and biologically meaningful spatial patterns of lesion distributions from stroke. The identified lesion atoms recapitulated well-known functional brain circuits. Moreover, the respective lesion patterns generally fit well with the associated functions engaged in the applied tasks, as evidenced by neuropsychological assessments of language, motor, memory, and attention, as well as cognitive and executive control (Duncan \& Owen, 2000; Fedorenko et al., 2013; Gregory Hickok \& Poeppel, 2007). The fine-grained nature of the extracted lesion atoms also enabled us to bring into sharp focus fiber pathways of essential relevance for the human language capacity. The implicated lesioned anatomical tracts recapitulated the dorsal and ventral route of the dual-stream concept of language processing (Gregory Hickok \& Poeppel, 2004, 2007), cerebral and cerebellar motor representations (Schmahmann et al., 2000), and visuo-spatial attention and executive control (De Schotten et al., 2011; Duncan \& Owen, 2000). Overall, our analyses on language performance show a stronger impact of lesions in the left hemisphere, conforming to the widely held asymmetry of the human language network (Hartwigsen et al., 2021).

The granularity of our lesion atoms further enabled us to dissect subcomponents of stroke effects in language-related pathways, especially the different perisylvian subsegments of the AF. Since 
the three-segment view of AF anatomy was proposed (Catani et al., 2005), various studies ventured to examine the role that subcomponents of the AF play in realizing language function. Such studies initially suggested that the functions of the distinct fiber bundle segments differ: the direct pathway was associated with phonological processes, whereas the indirect anterior segment tended to serve semantically-based functions, and the posterior segment could serve especially language comprehension (Catani et al., 2005). However, evidence from these studies have previously been hard to reconcile.

Some studies on aphasia after stroke linked the anterior segment of the left AF to impaired speech fluency, among other fiber tracts (Basilakos et al., 2014; Fridriksson et al., 2013). One study advocated significant language associations selectively for the posterior segment of AF (Ivanova et al., 2016). Yet, other investigators reported no significant links between the damage to the subsegments of the AF and subsequent language function (Q. Yu et al., 2019). Based on our investigation of a large clinical cohort, we provide newly detailed evidence that dissects the major white-matter pathways necessary for language production and understanding. In particular, our findings highlight the anterior and long segments as key subcomponents of the perisylvian route. Within the lesion atom of the dorsal pathway, both the anterior and long segments of the AF are further confirmed in the prediction of speech fluency in men and women. Indeed, our lesion atoms reflecting the left and right ventral language route further include the posterior segment of the AF. This finding suggests a shared essential piece of the dorsal and ventral language pathway and a contribution of the latter pathway to naming and semantic fluency deficits. Our observation ties into a previous lesion study which reported overlap between both streams in the left temporo-parietal region (Fridriksson et al., 2016). Going beyond a merely topographic interpretation, our study extends the previous findings by pointing to potentially shared underlying anatomical connections. Such an overlap seems plausible given that the associated language tasks, naming, and semantic fluency, likely invoke both fiber processing streams.

In both men and women, the strongest observed brain-behavior predictions were preferentially lateralized to the left hemisphere. We identified the fiber tracts of the left ILF, the IFOF, and the UF to be critically involved in naming and semantic fluency deficits in both sexes. The left AF was responsible for performance decline in phonemic fluency in women and men to a similar degree, which nicely mirrors earlier findings (Marchina et al., 2011; J. Wang et al., 2013). The involved anatomical tracts are in accord with incumbent theories around the dorsal and ventral language pathways (Gregory Hickok \& Poeppel, 2004, 2007; Saur et al., 2008). Our lesion-outcome effects in semantic fluency and naming were shared by women and men. This observation highlights the importance of the ventral language route and some of its major white matter pathways, including 
the ILF, IFOF, and UF. These findings directly converge with previous analyses that collapsed across both sexes into crude average effects. In particular, our finding linking semantic fluency with the ventral stream resonates well with vast evidence from large-scale neuroimaging meta-analyses (Binder et al., 2009; Vigneau et al., 2006), neurostimulation results in healthy participants (Pobric et al., 2007; Woollams, 2012), lesion studies (Bonilha et al., 2019; Fridriksson et al., 2016), computational modeling studies (Ueno et al., 2011), and targeted intraoperative electro-stimulation of fiber tracts (Duffau et al., 2005; Leclercq et al., 2010).

Our analyses detailed brain-behavior associations of phonemic fluency to locate to the dorsal perisylvian route, congruently for both sexes. With the strong impetus of the AF fiber tract, our results are consistent with the association of the dorsal language stream with phonological processing in healthy volunteers (Catani et al., 2005; Parker et al., 2005; Saur et al., 2008) and lesions in stroke patients (Fridriksson et al., 2016). Our predictive effects link the ventral and dorsal pathways to semantic and phonemic fluency, respectively. Both identified associations are aligned with findings from a variety of methodologies, including direct intraoperative electro-stimulation (Duffau et al., 2005; Leclercq et al., 2010), diffusion MRI tractography (Glasser \& Rilling, 2008; Parker et al., 2005), and lesion-symptom investigations in aphasia (Biesbroek et al., 2016; Butler et al., 2014; Faroqi-Shah et al., 2014; Kümmerer et al., 2013; Mirman et al., 2015; J. Zhang et al., 2018). We confirm the structural relations in the dual-stream model in regard to the underlying language tasks. Our lesion findings shared by women and men are hence consistent with previous studies that were blind to sex-specific effects.

More generally, several previous efforts compounded evidence that male and female brains are distinct in the asymmetry of functional brain organization. Male brains appear to show more far-reaching segregation between the left and right hemispheric function. Instead, female brains are thought to have stronger interconnections between the left and right hemispheres (Jaeger et al., 1998; Shaywitz et al., 1995). According to an early hypothesis, the sharper distinction between left and right brain function, allegedly characteristic for men, was thought to promote spatial but impede verbal performance (Levy, 1978; Shaywitz et al., 1995). On a cognitive level, this sex divergence in brain lateralization was thought to explain why women outperformed the opposite sex in language tasks such as verbal fluency tests on average; as well as why men were supposed to typically exhibit greater spatial skills, such as in three-dimensional mental rotation tasks (Broverman et al., 1972; Hyde, 2016). However, the respective effect sizes have been small and several meta-analyses called into question the organizational differences related to lateralization and language processing (Hirnstein et al., 2019; Ruigrok et al., 2014; Wallentin, 2009). 
In our sex-sensitive analyses, we found women to be more impaired by lesion damage in the left ventral pathway across different language tasks, including phonemic and semantic fluency. Moreover, we detailed a female-specific selective vulnerability to ventral lesion damage in phonemic fluency. Importantly, differences in lesion size or overall lesion topography were accounted for in our approach. A relatively stronger role of the left ventral pathway in women is in line with previous work in healthy volunteers, demonstrating higher radial diffusivity in the left UF in women relative to men (M. Jung et al., 2019).

In contrast, damage in the left-dominant ventral pathway and its right-hemispheric homolog was particularly informative about lost naming and semantic fluency capacities in men. Previous work suggested that the ventral language pathway is largely bilaterally organized, comprising parallel processing streams (Gregory Hickok \& Poeppel, 2004, 2007). We expand the current literature body by adding novel evidence for a predominantly left-lateralized ventral dominance in women. In support of our findings, early analyses stratified by sex suggested that men have a symmetrical increase in white matter volume and concentration in the bilateral anterior temporal lobe (Good et al., 2001). Our findings receive further support in that healthy male volunteers showed increased grey matter volume and cortical thickness in several bilateral regions relative to females, although no relationship with speech tests was reported (Angelopoulou et al., 2019; Ritchie et al., 2018). Notably, previous studies are inconclusive with respect to sex incongruencies in functional and structural substrates of language-related processes (Ullman et al., 2007; Xu et al., 2020). Our sex-aware analyses bear the potential to reconcile several important inconsistencies on structural lateralization effects in general and in the ventral language pathway in particular.

Indeed, our analyses brought into the open several sex-specific findings outside the canonical language circuits that shed new light on stroke-induced language deficits. Our sex-aware analysis framework offered evidence that men draw from bilateral ventral and cerebellar connections and potentially integrate more domain-general cognitive and executive functions via right-lateralized cingular fiber connections to enable efficient language processing after stroke. There is increasing evidence that many higher cognitive functions, including language processing, are supported by large-scale distributed networks (Braga et al., 2020; Fedorenko et al., 2013). Such parallel organization of networks may potentially aid recovery after aphasic stroke by the recruitment of other cognitive functions that compensate for the damaged language capacities (Brownsett et al., 2014). In particular, additional domain-general networks may be upregulated to boost the performance of the damaged processes if the primary language system is compromised (Geranmayeh et al., 2014, 2017; Stockert et al., 2020). Damage to cingular fiber tracts may potentially undermine the increased recruitment of domain-general networks, including the "multiple-demand network" with special 
contributions to cognitive control (Duncan \& Owen, 2000). The selective vulnerability we show for men in cingular pathways and bilateral temporal circuits may relate to previously identified sex-specific idiosyncracies in white matter microstructure in healthy subjects (Inano et al., 2011; Ritchie et al., 2018). The combination of core language network disruption and lesioned cingular pathways may hence reflect the complex balance of domain-specific and domain-general interplay for entertaining language functions.

In contrast, women accentuate left-dominant circuits, including structural connections of the fornix. A hippocampal-neocortical pathway through the fornix was previously suggested to be critical to the supra-modal brain network architecture of the default mode network (DMN, (Kernbach et al., 2018)). Large-scale associations networks, such as the DMN, fronto-parietal control, and salience networks, are generally thought to occupy regions juxtaposed and interdigitated with the language network (Braga et al., 2020; Margulies et al., 2016; Thomas Yeo et al., 2011). Altered supra-modal interactions with core language features via fornical fibers may hence reflect a dynamic process underlying neural remodeling in chronic aphasic stroke. Importantly, our findings capture the subacute phase of stroke; therefore the dynamic of reorganization may still change. Possibly, the altered language-DMN unit may affect language outcomes. Previous work has described disrupted DMN function in chronic stroke patients collapsed across sexes for impairment in motor function (Bonkhoff, Schirmer, Bretzner, Etherton, et al., 2021; Y. Zhang et al., 2017; Zhao et al., 2018) as well as language function (Balaev et al., 2016; Siegel et al., 2016; Tuladhar et al., 2013; C. Wang et al., 2014). Both the language-specific as well as domain-general disruption were suggested to influence post-stroke language outcome. However, the exact associations remain to be delineated.

Our findings speak in favor of revising the view towards sex-differentiated disruption of DMN integration. General sex divergences in DMN function in healthy individuals were previously demonstrated, indicating that women have higher connectivity within the DMN (Ritchie et al., 2018). The authors suggested that the higher intra-DMN connectivity may help explain the higher average female ability in different cognitive domains. The selective vulnerability to the fornix in women is further supported by a previous diffusion tensor investigation on healthy volunteers showing higher fractional anisotropy in women specifically in the hippocampus output pathway via the fornix (Inano et al., 2011). Moreover, in chronic stroke patients, the hippocampal-neocortical connectivity to the inferior parietal lobule (IPL) was consistently found to be decreased compared to healthy controls (J. Jung et al., 2021).

In fact, the IPL represents one potential hub for multi-sensory integration of domain-specific and supra-modal processes (Kernbach et al., 2018). Previous work has linked the IPL with semantic aspects of language processing, social cognition, and critical switch in stimulus-driven control of attention and diversion of self-reflective thinking to processing salient external stimuli (Bays et al., 
2010; Binder \& Desai, 2011; Bzdok et al., 2016; Kernbach et al., 2018). It receives axonal inputs from the posterior segment of the AF, as a short vertical tract connecting Wernicke's with Geschwind's areas (Catani et al., 2005). Our results further emphasize the relevance of the IPL and underlying posterior $\mathrm{AF}$ as seen in the dominance of the ventral lesion atom in women across all applied language tasks. Our findings converge with evidence suggesting negative modulatory connections from the parietal lobule to the superior temporal gyrus in females, enabling highly efficient semantic representations (Xu et al., 2020). Similarly, higher regional efficiency was reported in females in the left IPL and superior temporal regions in network connectivity analysis in neurologically intact participants (Gong et al., 2009).

Building on studies on healthy individuals, we confirm the relevance of the left IPL, fornix, and DMN in ischemic stroke patients. Our lesion-outcome findings in women may potentially reconcile previous observations and offer an explanation to sex divergences in chronic aphasia outcomes. More importantly, the personalized prediction of long-term post-stroke recovery using sex-aware stroke lesion topology may bring value to future individualized treatment interventions. Intensive speech therapy was previously shown to promote more efficient DMN integration leading to consequent language improvement (Abo et al., 2004; Dreyer et al., 2021; Marcotte et al., 2013; Musso et al., 1999). Targeted rehabilitation in men and women may hence advance therapy-induced neuroplasticity by enabling more efficient multimodal network integration. 


\section{Online Methods}

\section{Prospective stroke population resource}

We analyzed a multi-center stroke registry with 1,401 patients. They were retrospectively selected from the Bundang and Hallym Vascular Cognitive Impairment cohorts, which are prospectively recruited cohorts consisting of patients originally admitted to the Seoul National University Bundang Hospital or Hallym University Sacred Heart Hospital in South Korea between 2007 and 2018 (Kim et al., 2015). Patients were hospitalized and diagnosed with acute ischemic stroke. All patients were eligible for the present study based on the following criteria: (1) availability of brain MRI showing acute tissue infarction in the diffusion-weighted imaging (DWI) and/or fluid-attenuated inversion recovery (FLAIR), (2) successful lesion segmentation and registration, (3) no primary intracerebral hemorrhage, and (4) availability of follow-up data on key demographics and neuropsychological assessment (the 60-min Korean-Vascular Cognitive Impairment Harmonization Standards-Neuropsychology Protocol (Hachinski et al., 2006; K.-H. Yu et al., 2013)). The local institutional review boards of each hospital approved the study protocol and gave waived consent requirements based on the retrospective nature of this study and minimal risks to participants.

\section{Characteristics of stroke patient cohort}

The neuropsychological performance of the patients was profiled by a rich battery of tests $\sim 3$ months (Hallym: 91.4 days (SD 38.9), Bundang: 105.7 days (SD 94.1)) after the acute onset of stroke (K.-H. Yu et al., 2013). For the purpose of our present study, we primarily focused on two key facets of post-stroke language performance: i) naming ability (Korean short version of the Boston Naming Test (BN) (Kaplan et al., 1983)), and ii) speech fluency (phonemic and semantic fluency (PF, and SF, respectively; (K.-H. Yu et al., 2013)). The BN task prompts patients to name 15 pictured nouns (short version). In the semantic fluency tasks, patients are instructed to generate as many words as possible belonging to a specific category, such as animals, within a one minute time window. In the phonemic fluency task, in turn, patients are asked to generate words starting with a given phoneme (e.g., giut, iung, or siut, which correspond to the international phonemic alphabet phonemes of $\mathrm{g}, \mathrm{y}$, and $\mathrm{s}$, respectively). These fluency tests have been previously validated in the Korean language (Kang et al., 2000). A diverse collection of general cognitive tests included the Korean Mini-Mental State Examination (MMSE), the Seoul Verbal Learning test (SVLT), the Rey Complex Figures test (RCFT), Digital symbol coding, and the Korean-Trail Making Tests Elderly's version A and B (TMT). The Informant Questionnaire on Cognitive Decline in the Elderly (IQCODE) captured the cognitive performance before the ischemic event (D. W. Lee et al., 2005). Available sociodemographic and 
clinical information included age, sex, years of education, and lesion volume (Table 1). All continuous variables were adjusted to a comparable scale by mean centering to zero and variance scaling to one.

\section{Neuroimaging data preprocessing}

Whole-brain MRI scans were typically acquired within the first week after the stroke event. The scanning protocol included structural axial T1, T2-weighted spin-echo, FLAIR, and DWI sequences (3.0T, Achieva scanner, Philips Healthcare, Netherlands; cf., supplementary materials for details). Tissue damage was manually segmented on DWI or less frequently FLAIR images by experienced investigators (A.K.K. and G.A.) relying on in-house developed software based on MeVisLab (MeVis Medical Solutions AG, Bremen, Germany; (Ritter et al., 2011)). Lesion segmentations were checked and potentially refined by two additional experienced raters. Brain scans and their corresponding lesion segmentations were linearly and nonlinearly normalized to Montreal Neurological Institute (MNI-152) reference space employing the publicly available RegLSM image processing pipeline (Weaver et al., 2019). Quality of normalization was rigorously controlled by an experienced rater and if necessary manually corrected.

\section{Data-led deconvolution of hidden lesion atoms}

We sought to explore the coherent patterns that may combine to make up the topographical distribution of stroke lesions in white matter. First, we parsed each patient's lesion fingerprint by summarizing the white-matter damage annotations at the voxel level according to fiber tract definitions from a reference tractography atlas (Catani \& Thiebaut de Schotten, 2008; Thiebaut de Schotten et al., 2011). This widely-used atlas provided spatial definitions of 28 major fiber tracts in MNI standard space (Assaneo et al., 2019; Corbetta et al., 2015; Fridriksson et al., 2013; Lunven et al., 2015; Zeestraten et al., 2017). In doing so, the tract-by-tract summaries of lesion loads separately tracked the left and right hemispheres, which enabled the modeling of subtle differences in hemispheric lateralization in downstream analysis steps (Catani et al., 2007; Thiebaut de Schotten et al., 2011).

Of particular relevance to the present investigation due to their roles in human-defining language capacity, we captured the neuroanatomy of the core perisylvian circuitry by the canonical arcuate fasciculus (AF), subdivided into i) long, ii) anterior, and iii) posterior segments of the tract (Catani et al., 2005; Catani \& Mesulam, 2008; Catani \& Thiebaut de Schotten, 2008; Dick \& Tremblay, 2012). The ventral pathway consisted of the uncinate fasciculus (UF), the inferior longitudinal fasciculus (IFL), and the inferior fronto-occipital fasciculus (IFOF) (Axer et al., 2013; 
Catani et al., 2003; Saur et al., 2008; Vigneau et al., 2006). Limbic pathways included the fornix and cingular white-matter bundles (Catani \& Thiebaut de Schotten, 2008). Motor projections were taken into account by the internal capsule (IC), the corticospinal tract (CST), and cerebellar connections, including the cortico-pontine-cerebellar tract (CPC) and the inferior and superior cerebellar peduncle (ICP, SCP, respectively). The topography of atlas fiber tracts directly informed the labeling of the derived lesion atoms. For consistency, the resulting lesion atoms of the language system were labeled according to widely acknowledged definitions of the dual-stream model of language (Gregory Hickok \& Poeppel, 2004). We $\log _{2}$-transformed and concatenated the measures of each of the 28 tract measures of lesion loads for all 1,401 patients. Once each tract lesion load (Marchina et al., 2011; J. Wang et al., 2013) was recorded in each patient, we carried out non-negative matrix factorization (NNMF) as a multivariate pattern discovery strategy (D. D. Lee \& Seung, 1999). This unsupervised machine-learning algorithm can identify the topological form and patient-specific combination of lesion pattern expressions that make up the white matter disconnection inflicted by stroke. In accordance with our recent stroke studies (Bonkhoff, Lim, Bae, Weaver, Kuijf, et al., 2021; Bonkhoff, Schirmer, Bretzner, Hong, et al., 2021), the derived sum-of-parts representation was henceforth referred to as lesion atoms.

More formally, NNMF achieves a low-rank approximation of the data $V$, with $V$ reflecting the lesion load summaries of 28 tracts, with dimensions $m \times n$ ( $m=$ number of tracts in the atlas, $n=$ number of patients), by partitioning the interindividual variation in white-matter strokes into a matrix $W$ of $k$ part-based factor representations. The latent pattern expression matrix $H$ indicated how relevant each emerging lesion atom is to describe the constituent parts of an individual patient's overall brain lesion distribution. $W$ and $H$ thus carried $m \times k$ and $k \times n$ dimensions, respectively. Given by $V=W H$, the latent factorization hence deconvolved the actual lesion constellation in a particular patient into a parts-based representation.

In contrast to alternative dimensionality reduction tools, NNMF provided at least two important advantages for the goal of the present study. First, the non-negative value encoding of the differential white-matter involvement and the inherent non-negativity constraint of the NNMF algorithm allows for intuitive neurobiologically meaningful interpretation. For each patient $j$, the latent pattern expression $H_{j}$ offered a directly interpretable model of the lesion variation across patients in our cohort. In contrast, alternative matrix factorization algorithms, such as principal component analysis, typically recover patterns by incurring various cancellations between positive and negative pattern loadings. Such alternative treatment of the derived hidden parts-of-whole representations would have hurt intuitive neurobiological meaning. By means of strictly additive combinations of pattern contributions (allowing for no subtractions), NNMF allows for biologically more meaningful and interpretable representations of lesion constellations. Second, classical clustering approaches for dimensionality reduction would consider the effect of each differential tract involvement once only. In contrast, by embracing NNMF, damage to each white-matter tract in the 
reference atlas has the opportunity to belong to multiple latent factors of $W$ in continuous degrees. In this way, each tract lesion load could play a distinct role in different lesion atoms, each of which reflected extracted lesion-specific topological motifs distributed across the whole white matter.

\section{Predicting language outcome at 3 months post-stroke from patient-specific lesion fingerprints}

The thus derived lesion atoms served as a low-dimensional summary of the individual lesion fingerprints. Expression degrees of these lesion patterns, specific to each particular stroke patient, provided the input to our Bayesian model (Gelman \& Hill, 2006) to explain the interindividual differences in language performance outcomes at $\sim 3$ months after the stroke event. We designed a Bayesian generative modeling tactic. We aimed to obtain fully probabilistic parameter estimates that could inform us about effect strength and effect certainty of how each particular lesion pattern is responsible for the clinical outcome. To place a special focus on the putative effects of sex divergences on post-stroke language performance, we introduced dedicated model parameters, which allowed to estimate each pattern regression slope in a sex-specific manner. That is, we could estimate the overlapping and distinct roles of each lesion atom in tracking cognitive facets of language in women and men separately within an integrated modeling solution. The model structure was as follows:

$$
\begin{aligned}
& \alpha \sim \operatorname{Normal}(\mu=0, \sigma=1) \\
& \beta_{1-10} \sim \operatorname{Normal}(\mu=0, \sigma=1)_{[\text {[sex] }} \\
& \beta_{\text {lesion volume }} \sim \operatorname{Normal}(\mu=0, \sigma=1) \\
& \beta_{\text {age }} \sim \operatorname{Normal}(\mu=0, \sigma=10) \\
& \beta_{\text {age }{ }^{*} \text { age }} \sim \operatorname{Normal}(\mu=0, \sigma=10) \\
& \beta_{\text {education years }} \sim \operatorname{Normal}(\mu=0, \sigma=5) \\
& \beta_{\text {IQCODE }} \sim \operatorname{Normal}(\mu=0, \sigma=1) \\
& \beta_{\text {male }} \sim \operatorname{Normal}(\mu=0, \sigma=1) \\
& \beta_{\text {female }} \sim \operatorname{Normal}(\mu=0, \sigma=1) \\
& \text { cognitive_outcome }=\alpha+\beta_{1[\mathrm{sex}]} * \text { Lesion atom }_{1}+\ldots+\beta_{10[\mathrm{sex}]} * \text { Lesion atom }_{10} \\
& +\beta_{\text {lesion volume }} * \text { Lesion volume }+\beta_{\text {age }} * \text { Age }+\beta_{\text {age*age }} * \text { Age }^{2}+\beta_{\text {education years }} * \text { Education years } \\
& +\beta_{\mathrm{IQCODE}} * \mathrm{IQCODE}+\beta_{\text {male }} * \text { Male }+\beta_{\text {female }} * \text { Female }
\end{aligned}
$$


A separate probabilistic Bayesian model was estimated for each dedicated post-stroke performance score in each of the target language domains: BN, SF, and PF (cf. above; for other cognitive assessments see Supplement). All analyses were appropriately adjusted for variation that could be explained by the covariates age, age ${ }^{2}$, sex, education in years, pre-morbid cognitive performance, and total lesion volume. We adjusted for age-specific effects by considering the bare age and its squared value and, therefore, captured possible u-shaped effects. Our parametric model specification also included sex as a covariate to take into account outcome differences that were independent and dependent of the lesion configurations captured in the NNMF-derived lesion atom representations (see (Bonkhoff, Lim, Bae, Weaver, Kuijf, et al., 2021; Bonkhoff, Schirmer, Bretzner, Hong, et al., 2021)). Samples from the posterior distribution of the model parameters were drawn by the No U-Turn Sampler (NUTS, (Hoffman et al., 2014)), a kind of Monte Carlo Markov Chain algorithm (in our setting defaulted to draws $=5,000$ ).

To evaluate the practical usefulness of the thus inferred posterior parameter distributions, we computed posterior predictive checks for every analysis of a given language performance outcome. These model-based simulations of new data were then compared to the observed data to determine the predictive performance measured as coefficient of determination $\left(\mathrm{R}^{2}\right)$. That is, we assessed the model-simulated outcome predictions generated by our Bayesian model to approximate an external validation based on our patient cohort. This empirical procedure is a well-recognized option for judging the adequacy of Bayesian models given the actual data at hand (Gelman et al., 2013; Kruschke, 2014).

\section{Code availability}

Data analyses were conducted in a Python 3.8.5 (IPython 7.21.0) environment and primarily relied on the packages nilearn, scikit-learn, and pymc3 (Abraham et al., 2014; Salvatier et al., 2016). Full code is accessible to and open for reuse by the reader here: http://github.com/jkernbach/to_be_added_later. The probabilistic tractography atlas is available at: https://www.natbrainlab.co.uk/atlas-maps. The use of dynamic reporting guarantees full reproducibility of the results. 
bioRxiv preprint doi: https://doi.org/10.1101/2022.01.18.474989; this version posted January 18,2022 . The copyright holder for this preprint (which was not certified by peer review) is the author/funder, who has granted bioRxiv a license to display the preprint in perpetuity. It is made available under aCC-BY-NC-ND 4.0 International license. 


\section{Acknowledgments \& Disclosure}

We confirm that we have read the Journal's position on issues involved in ethical publication and affirm that this report is consistent with those guidelines.

We gratefully acknowledge Angelina K. Kancheva and Gözdem Arikan for their help with performing the manual infarct segmentations, Jae-seol Park, and Eunbin Ko for their help in organizing the neuropsychological data, and Parashkev Nachev and Michel Thiebaut de Schotten for their valuable comments on earlier versions of the manuscript.

Funding: This project has been made possible by the Brain Canada Foundation, through the Canada Brain Research Fund, with the financial support of Health Canada, National Institutes of Health (NIH R01 AG068563A), and the Canadian Institute of Health Research (CIHR 438531). DB was also supported by the Healthy Brains Healthy Lives initiative (Canada First Research Excellence fund), Google (Research Award), and by the CIFAR Artificial Intelligence Chairs program (Canada Institute for Advanced Research).

Conflicts of interest/Competing interests: None of the authors has any conflict of interest to disclose.

Availability of data and code: Additional data is made available in the supplemental methods and supplemental results online. 


\section{References}

Abo, M., Senoo, A., Watanabe, S., Miyano, S., Doseki, K., Sasaki, N., Kobayashi, K., Kikuchi, Y., \& Yonemoto, K. (2004). Language-related brain function during word repetition in post-stroke aphasics. Neuroreport, 15(12), 1891-1894.

Abraham, A., Pedregosa, F., Eickenberg, M., Gervais, P., Mueller, A., Kossaifi, J., Gramfort, A., Thirion, B., \& Varoquaux, G. (2014). Machine learning for neuroimaging with scikit-learn. Frontiers in Neuroinformatics, 8,14 .

Angelopoulou, G., Meier, E. L., Kasselimis, D., Pan, Y., Tsolakopoulos, D., Velonakis, G., Karavasilis, E., Kelekis, N. L., Goutsos, D., Potagas, C., \& Kiran, S. (2019). Investigating Gray and White Matter Structural Substrates of Sex Differences in the Narrative Abilities of Healthy Adults. Frontiers in Neuroscience, 13, 1424.

Assaneo, M. F., Ripollés, P., Orpella, J., Lin, W. M., de Diego-Balaguer, R., \& Poeppel, D. (2019). Spontaneous synchronization to speech reveals neural mechanisms facilitating language learning. Nature Neuroscience, 22(4), 627-632.

Axer, H., Klingner, C. M., \& Prescher, A. (2013). Fiber anatomy of dorsal and ventral language streams. Brain and Language, 127(2), 192-204.

Balaev, V., Petrushevsky, A., \& Martynova, O. (2016). Changes in Functional Connectivity of Default Mode Network with Auditory and Right Frontoparietal Networks in Poststroke Aphasia. Brain Connectivity, 6(9), 714-723.

Baldo, J. V., Arévalo, A., Patterson, J. P., \& Dronkers, N. F. (2013). Grey and white matter correlates of picture naming: evidence from a voxel-based lesion analysis of the Boston Naming Test. Cortex; a Journal Devoted to the Study of the Nervous System and Behavior, 49(3), 658-667.

Basilakos, A., Fillmore, P. T., Rorden, C., Guo, D., Bonilha, L., \& Fridriksson, J. (2014). Regional white matter damage predicts speech fluency in chronic post-stroke aphasia. Frontiers in Human Neuroscience, 8, 845.

Basilakos, A., Rorden, C., Bonilha, L., Moser, D., \& Fridriksson, J. (2015). Patterns of poststroke brain damage that predict speech production errors in apraxia of speech and aphasia dissociate. Stroke; a Journal of Cerebral Circulation, 46(6), 1561-1566.

Bates, E., Wilson, S. M., Saygin, A. P., Dick, F., Sereno, M. I., Knight, R. T., \& Dronkers, N. F. (2003). Voxel-based lesion-symptom mapping. Nature Neuroscience, 6(5), 448-450.

Bays, P. M., Singh-Curry, V., Gorgoraptis, N., Driver, J., \& Husain, M. (2010). Integration of goal- and stimulus-related visual signals revealed by damage to human parietal cortex. The Journal of Neuroscience: The Official Journal of the Society for Neuroscience, 30(17), 5968-5978.

Biesbroek, J. M., van Zandvoort, M. J. E., Kappelle, L. J., Velthuis, B. K., Biessels, G. J., \& Postma, A. (2016). Shared and distinct anatomical correlates of semantic and phonemic fluency revealed by lesion-symptom mapping in patients with ischemic stroke. Brain Structure \& Function, 221(4), 2123-2134.

Binder, J. R., \& Desai, R. H. (2011). The neurobiology of semantic memory. Trends in Cognitive Sciences, 15(11), 527-536.

Binder, J. R., Desai, R. H., Graves, W. W., \& Conant, L. L. (2009). Where is the semantic system? A critical review and meta-analysis of 120 functional neuroimaging studies. Cerebral Cortex , 19(12), 2767-2796.

Bonilha, L., Hillis, A. E., Hickok, G., den Ouden, D. B., Rorden, C., \& Fridriksson, J. (2017). Temporal lobe networks supporting the comprehension of spoken words. Brain: A Journal of Neurology, 140(9), $2370-2380$.

Bonilha, L., Hillis, A. E., Wilmskoetter, J., Hickok, G., Basilakos, A., Munsell, B., Rorden, C., \& Fridriksson, J. (2019). Neural structures supporting spontaneous and assisted (entrained) speech fluency. Brain: A Journal of Neurology, 142(12), 3951-3962.

Bonkhoff, A. K., Karch, A., Weber, R., Wellmann, J., \& Berger, K. (2021). Female Stroke: Sex Differences in Acute Treatment and Early Outcomes of Acute Ischemic Stroke. Stroke; a Journal of Cerebral Circulation, 52(2), 406-415.

Bonkhoff, A. K., Lim, J.-S., Bae, H.-J., Weaver, N. A., Kuijf, H. J., Biesbroek, J. M., Rost, N. S., \& Bzdok, D. (2021). Generative lesion pattern decomposition of cognitive impairment after stroke. Brain Communications, 3(2), fcab110. 
Bonkhoff, A. K., Schirmer, M. D., Bretzner, M., Etherton, M., Donahue, K., Tuozzo, C., Nardin, M., Giese, A.-K., Wu, O., D Calhoun, V., Grefkes, C., \& Rost, N. S. (2021). Abnormal dynamic functional connectivity is linked to recovery after acute ischemic stroke. Human Brain Mapping, 42(7), 2278-2291.

Bonkhoff, A. K., Schirmer, M. D., Bretzner, M., Hong, S., Regenhardt, R. W., Brudfors, M., Donahue, K. L., Nardin, M. J., Dalca, A. V., Giese, A.-K., Etherton, M. R., Hancock, B. L., Mocking, S. J. T., McIntosh, E. C., Attia, J., Benavente, O. R., Bevan, S., Cole, J. W., Donatti, A., ... Rost, N. S. (2021). Outcome after acute ischemic stroke is linked to sex-specific lesion patterns. Nature Communications, 12(1), 3289.

Braga, R. M., DiNicola, L. M., Becker, H. C., \& Buckner, R. L. (2020). Situating the left-lateralized language network in the broader organization of multiple specialized large-scale distributed networks. Journal of Neurophysiology, 124(5), 1415-1448.

Broverman, I. K., Vogel, S. R., Broverman, D. M., Clarkson, F. E., \& Rosenkrantz, P. S. (1972). Sex-role stereotypes: A current appraisal. The Journal of Social Issues, 28(2), 59-78.

Brownsett, S. L. E., Warren, J. E., Geranmayeh, F., Woodhead, Z., Leech, R., \& Wise, R. J. S. (2014). Cognitive control and its impact on recovery from aphasic stroke. Brain: A Journal of Neurology, 137(Pt 1), 242-254.

Bushnell, C., McCullough, L. D., Awad, I. A., Chireau, M. V., Fedder, W. N., Furie, K. L., Howard, V. J., Lichtman, J. H., Lisabeth, L. D., Piña, I. L., Reeves, M. J., Rexrode, K. M., Saposnik, G., Singh, V., Towfighi, A., Vaccarino, V., Walters, M. R., American Heart Association Stroke Council, Council on Cardiovascular and Stroke Nursing, ... Council for High Blood Pressure Research. (2014). Guidelines for the prevention of stroke in women: a statement for healthcare professionals from the American Heart Association/American Stroke Association. Stroke; a Journal of Cerebral Circulation, 45(5), 1545-1588.

Butler, R. A., Lambon Ralph, M. A., \& Woollams, A. M. (2014). Capturing multidimensionality in stroke aphasia: mapping principal behavioural components to neural structures. Brain: A Journal of Neurology, 137(Pt 12), 3248-3266.

Button, K. S., Ioannidis, J. P. A., Mokrysz, C., Nosek, B. A., Flint, J., Robinson, E. S. J., \& Munafò, M. R. (2013). Power failure: why small sample size undermines the reliability of neuroscience. Nature Reviews. Neuroscience, 14(5), 365-376.

Bzdok, D., Hartwigsen, G., Reid, A., Laird, A. R., Fox, P. T., \& Eickhoff, S. B. (2016). Left inferior parietal lobe engagement in social cognition and language. Neuroscience and Biobehavioral Reviews, 68, 319-334.

Cahill, L. (2006). Why sex matters for neuroscience. Nature Reviews. Neuroscience, 7(6), 477-484.

Catani, M., Allin, M. P. G., Husain, M., Pugliese, L., Mesulam, M. M., Murray, R. M., \& Jones, D. K. (2007). Symmetries in human brain language pathways correlate with verbal recall. Proceedings of the National Academy of Sciences of the United States of America, 104(43), 17163-17168.

Catani, M., Jones, D. K., Donato, R., \& Ffytche, D. H. (2003). Occipito-temporal connections in the human brain. Brain: A Journal of Neurology, 126(9), 2093-2107.

Catani, M., Jones, D. K., \& Ffytche, D. H. (2005). Perisylvian language networks of the human brain. Annals of Neurology, 57(1), 8-16.

Catani, M., \& Mesulam, M. (2008). The arcuate fasciculus and the disconnection theme in language and aphasia: History and current state. Cortex; a Journal Devoted to the Study of the Nervous System and Behavior, 44(8), 953-961.

Catani, M., \& Thiebaut de Schotten, M. (2008). A diffusion tensor imaging tractography atlas for virtual in vivo dissections. Cortex; a Journal Devoted to the Study of the Nervous System and Behavior, 44(8), 1105-1132.

Corbetta, M., Ramsey, L., Callejas, A., Baldassarre, A., Hacker, C. D., Siegel, J. S., Astafiev, S. V., Rengachary, J., Zinn, K., Lang, C. E., Connor, L. T., Fucetola, R., Strube, M., Carter, A. R., \& Shulman, G. L. (2015). Common behavioral clusters and subcortical anatomy in stroke. Neuron, 85(5), 927-941.

Dehlendorff, C., Andersen, K. K., \& Olsen, T. S. (2015). Sex Disparities in Stroke: Women Have More Severe Strokes but Better Survival Than Men. Journal of the American Heart Association, 4(7). https://doi.org/10.1161/JAHA.115.001967

Dejerine, J., Dejerine, J., \& Dejerine, A. (1895). Anatomie des centres nerveux. Rueff.

De Schotten, M. T., Dell'Acqua, F., Forkel, S., Simmons, A., Vergani, F., Murphy, D. G. M., \& Catani, M. (2011). A lateralized brain network for visuo-spatial attention. Nature Precedings, 1-1. 
Di Carlo, A., Lamassa, M., Baldereschi, M., Pracucci, G., Basile, A. M., Wolfe, C. D. A., Giroud, M., Rudd, A., Ghetti, A., Inzitari, D., \& European BIOMED Study of Stroke Care Group. (2003). Sex differences in the clinical presentation, resource use, and 3-month outcome of acute stroke in Europe: data from a multicenter multinational hospital-based registry. Stroke; a Journal of Cerebral Circulation, 34(5), 1114-1119.

Dick, A. S., \& Tremblay, P. (2012). Beyond the arcuate fasciculus: consensus and controversy in the connectional anatomy of language. Brain: A Journal of Neurology, 135(Pt 12), 3529-3550.

Dreyer, F. R., Doppelbauer, L., Büscher, V., Arndt, V., Stahl, B., Lucchese, G., Hauk, O., Mohr, B., \& Pulvermüller, F. (2021). Increased Recruitment of Domain-General Neural Networks in Language Processing Following Intensive Language-Action Therapy: fMRI Evidence From People With Chronic Aphasia. American Journal of Speech-Language Pathology / American Speech-Language-Hearing Association, 30(1S), 455-465.

Duffau, H., Gatignol, P., Mandonnet, E., Peruzzi, P., Tzourio-Mazoyer, N., \& Capelle, L. (2005). New insights into the anatomo-functional connectivity of the semantic system: a study using cortico-subcortical electrostimulations. Brain: A Journal of Neurology, 128(Pt 4), 797-810.

Duncan, J., \& Owen, A. M. (2000). Common regions of the human frontal lobe recruited by diverse cognitive demands. Trends in Neurosciences, 23(10), 475-483.

Eichert, N., Verhagen, L., Folloni, D., Jbabdi, S., Khrapitchev, A. A., Sibson, N. R., Mantini, D., Sallet, J., \& Mars, R. B. (2019). What is special about the human arcuate fasciculus? Lateralization, projections, and expansion. Cortex; a Journal Devoted to the Study of the Nervous System and Behavior, 118, 107-115.

Engelter, S. T., Gostynski, M., Papa, S., Frei, M., Born, C., Ajdacic-Gross, V., Gutzwiller, F., \& Lyrer, P. A. (2006). Epidemiology of aphasia attributable to first ischemic stroke: incidence, severity, fluency, etiology, and thrombolysis. Stroke; a Journal of Cerebral Circulation, 37(6), 1379-1384.

Faroqi-Shah, Y., Kling, T., Solomon, J., Liu, S., Park, G., \& Braun, A. (2014). Lesion analysis of language production deficits in aphasia. Aphasiology, 28(3), 258-277.

Fedorenko, E., Duncan, J., \& Kanwisher, N. (2013). Broad domain generality in focal regions of frontal and parietal cortex. Proceedings of the National Academy of Sciences of the United States of America, 110(41), 16616-16621.

Feigin, V. L., Forouzanfar, M. H., Krishnamurthi, R., Mensah, G. A., Connor, M., Bennett, D. A., Moran, A. E., Sacco, R. L., Anderson, L., Truelsen, T., O’Donnell, M., Venketasubramanian, N., Barker-Collo, S., Lawes, C. M. M., Wang, W., Shinohara, Y., Witt, E., Ezzati, M., Naghavi, M., \& Murray, C. (2014). Global and regional burden of stroke during 1990-2010: findings from the Global Burden of Disease Study 2010. The Lancet, 383(9913), 245-255.

Feigin, V. L., Norrving, B., \& Mensah, G. A. (2017). Global Burden of Stroke. Circulation Research, 120(3), 439-448.

Fridriksson, J., Guo, D., Fillmore, P., Holland, A., \& Rorden, C. (2013). Damage to the anterior arcuate fasciculus predicts non-fluent speech production in aphasia. Brain: A Journal of Neurology, 136(Pt 11), 3451-3460.

Fridriksson, J., Yourganov, G., Bonilha, L., Basilakos, A., Den Ouden, D.-B., \& Rorden, C. (2016). Revealing the dual streams of speech processing. Proceedings of the National Academy of Sciences of the United States of America, 113(52), 15108-15113.

Gelman, A., Carlin, J. B., Stern, H. S., Dunson, D. B., Vehtari, A., \& Rubin, D. B. (2013). Bayesian Data Analysis. Chapman and Hall/CRC.

Gelman, A., \& Hill, J. (2006). Data Analysis Using Regression and Multilevel/Hierarchical Models. Cambridge University Press.

Geranmayeh, F., Brownsett, S. L. E., \& Wise, R. J. S. (2014). Task-induced brain activity in aphasic stroke patients: what is driving recovery? Brain: A Journal of Neurology, 137(Pt 10), 2632-2648.

Geranmayeh, F., Chau, T. W., Wise, R. J. S., Leech, R., \& Hampshire, A. (2017). Domain-general subregions of the medial prefrontal cortex contribute to recovery of language after stroke. Brain: A Journal of Neurology, 140(7), 1947-1958.

Geschwind, N. (1970). The organization of language and the brain. Science, 170(3961), 940-944.

Gialanella, B., \& Prometti, P. (2009). Rehabilitation length of stay in patients suffering from aphasia after stroke. Topics in Stroke Rehabilitation, 16(6), 437-444. 
Giroud, M., Delpont, B., Daubail, B., Blanc, C., Durier, J., Giroud, M., \& Béjot, Y. (2017). Temporal trends in sex differences with regard to stroke incidence: The Dijon stroke registry (1987-2012). Stroke; a Journal of Cerebral Circulation, 48(4), 846-849.

Glasser, M. F., \& Rilling, J. K. (2008). DTI tractography of the human brain's language pathways. Cerebral Cortex , 18(11), 2471-2482.

Gong, G., Rosa-Neto, P., Carbonell, F., Chen, Z. J., He, Y., \& Evans, A. C. (2009). Age- and gender-related differences in the cortical anatomical network. The Journal of Neuroscience: The Official Journal of the Society for Neuroscience, 29(50), 15684-15693.

Good, C. D., Johnsrude, I., Ashburner, J., Henson, R. N., Friston, K. J., \& Frackowiak, R. S. (2001). Cerebral asymmetry and the effects of sex and handedness on brain structure: a voxel-based morphometric analysis of 465 normal adult human brains. NeuroImage, 14(3), 685-700.

Hachinski, V., Iadecola, C., Petersen, R. C., Breteler, M. M., Nyenhuis, D. L., Black, S. E., Powers, W. J., DeCarli, C., Merino, J. G., Kalaria, R. N., Vinters, H. V., Holtzman, D. M., Rosenberg, G. A., Wallin, A., Dichgans, M., Marler, J. R., \& Leblanc, G. G. (2006). National Institute of Neurological Disorders and Stroke-Canadian Stroke Network Vascular Cognitive Impairment Harmonization Standards. In Stroke (Vol. 37, Issue 9, pp. 2220-2241). https://doi.org/10.1161/01.str.0000237236.88823.47

Hartwigsen, G., Bengio, Y., \& Bzdok, D. (2021). How does hemispheric specialization contribute to human-defining cognition? Neuron, 109(13), 2075-2090.

Harvey, D. Y., \& Schnur, T. T. (2015). Distinct loci of lexical and semantic access deficits in aphasia: Evidence from voxel-based lesion-symptom mapping and diffusion tensor imaging. Cortex; a Journal Devoted to the Study of the Nervous System and Behavior, 67, 37-58.

Hickok, G. (2009). The functional neuroanatomy of language. Physics of Life Reviews, 6(3), 121-143.

Hickok, G., \& Poeppel, D. (2000). Towards a functional neuroanatomy of speech perception. Trends in Cognitive Sciences, 4(4), 131-138.

Hickok, G., \& Poeppel, D. (2004). Dorsal and ventral streams: a framework for understanding aspects of the functional anatomy of language. Cognition, 92(1-2), 67-99.

Hickok, G., \& Poeppel, D. (2007). The cortical organization of speech processing. Nature Reviews. Neuroscience, 8(5), 393-402.

Hier, D. B., Yoon, W. B., Mohr, J. P., Price, T. R., \& Wolf, P. A. (1994). Gender and aphasia in the Stroke Data Bank. Brain and Language, 47(1), 155-167.

Hirnstein, M., Hugdahl, K., \& Hausmann, M. (2019). Cognitive sex differences and hemispheric asymmetry: A critical review of 40 years of research. Laterality, 24(2), 204-252.

Hoffman, M. D., Gelman, A., \& Others. (2014). The No-U-Turn sampler: adaptively setting path lengths in Hamiltonian Monte Carlo. Journal of Machine Learning Research: JMLR, 15(1), 1593-1623.

Holroyd-Leduc, J. M., Kapral, M. K., Austin, P. C., \& Tu, J. V. (2000). Sex differences and similarities in the management and outcome of stroke patients. Stroke; a Journal of Cerebral Circulation, 31(8), 1833-1837.

Hyde, J. S. (2016). Sex and cognition: gender and cognitive functions. Current Opinion in Neurobiology, 38, $53-56$

Inano, S., Takao, H., Hayashi, N., Abe, O., \& Ohtomo, K. (2011). Effects of age and gender on white matter integrity. AJNR. American Journal of Neuroradiology, 32(11), 2103-2109.

Ito, K. L., \& Liew, S.-L. (2016). Calculating the laterality index using FSL for stroke neuroimaging data. GigaScience, 5(suppl_1), s13742-016.

Ivanova, M. V., Isaev, D. Y., Dragoy, O. V., Akinina, Y. S., Petrushevskiy, A. G., Fedina, O. N., Shklovsky, V. M., \& Dronkers, N. F. (2016). Diffusion-tensor imaging of major white matter tracts and their role in language processing in aphasia. Cortex; a Journal Devoted to the Study of the Nervous System and Behavior, 85, 165-181.

Jaeger, J. J., Lockwood, A. H., Van Valin, R. D., Kemmerer, D. L., Murphy, B. W., \& Wack, D. S. (1998). Sex differences in brain regions activated by grammatical and reading tasks. In NeuroReport (Vol. 9, Issue 12, pp. 2803-2807). https://doi.org/10.1097/00001756-199808240-00022

Joel, D., Berman, Z., Tavor, I., Wexler, N., Gaber, O., Stein, Y., Shefi, N., Pool, J., Urchs, S., Margulies, D. S., Liem, F., Hänggi, J., Jäncke, L., \& Assaf, Y. (2015). Sex beyond the genitalia: The human brain mosaic. Proceedings of the National Academy of Sciences of the United States of America, 112(50), 15468-15473. 
Jung, J., Laverick, R., Nader, K., Brown, T., Morris, H., Wilson, M., Auer, D. P., Rotshtein, P., \& Hosseini, A. A. (2021). Altered hippocampal functional connectivity patterns in patients with cognitive impairments following ischaemic stroke: A resting-state fMRI study. NeuroImage. Clinical, 32, 102742.

Jung, M., Mody, M., Fujioka, T., Kimura, Y., Okazawa, H., \& Kosaka, H. (2019). Sex Differences in White Matter Pathways Related to Language Ability. Frontiers in Neuroscience, 13, 898.

Kang, Y., Chin, J.-H., Na, D. L., Lee, J., \& Park, J. S. (2000). A normative study of the Korean version of Controlled Oral Word Association Test (COWAT) in the elderly. Korean Journal of Clinical Psychology, 19(2), 385-392.

Kaplan, E., Goodglass, H., \& Weintraub, S. (1983). The Boston Naming Test. Lea \& Febiger. Philadelphia, PA.

Kernbach, J. M., Yeo, B. T. T., Smallwood, J., Margulies, D. S., Thiebaut de Schotten, M., Walter, H., Sabuncu, M. R., Holmes, A. J., Gramfort, A., Varoquaux, G., Thirion, B., \& Bzdok, D. (2018). Subspecialization within default mode nodes characterized in 10,000 UK Biobank participants. Proceedings of the National Academy of Sciences of the United States of America, 115(48), 12295-12300.

Kiesow, H., Uddin, L. Q., Bernhardt, B. C., Kable, J., \& Bzdok, D. (2021). Dissecting the midlife crisis: disentangling social, personality and demographic determinants in social brain anatomy. Communications Biology, 4(1), 728.

Kim, B. J., Park, J.-M., Kang, K., Lee, S. J., Ko, Y., Kim, J. G., Cha, J.-K., Kim, D.-H., Nah, H.-W., Han, M.-K., Park, T. H., Park, S.-S., Lee, K. B., Lee, J., Hong, K.-S., Cho, Y.-J., Lee, B.-C., Yu, K.-H., Oh, M.-S., ... Bae, H.-J. (2015). Case characteristics, hyperacute treatment, and outcome information from the clinical research center for stroke-fifth division registry in South Korea. Journal of Stroke and Cerebrovascular Diseases: The Official Journal of National Stroke Association, 17(1), 38-53.

Kruschke, J. (2014). Doing Bayesian Data Analysis: A Tutorial with R, JAGS, and Stan. Academic Press.

Kümmerer, D., Hartwigsen, G., Kellmeyer, P., Glauche, V., Mader, I., Klöppel, S., Suchan, J., Karnath, H.-O., Weiller, C., \& Saur, D. (2013). Damage to ventral and dorsal language pathways in acute aphasia. Brain: A Journal of Neurology, 136(Pt 2), 619-629.

Laska, A. C., Hellblom, A., Murray, V., Kahan, T., \& Von Arbin, M. (2001). Aphasia in acute stroke and relation to outcome. Journal of Internal Medicine, 249(5), 413-422.

Leclercq, D., Duffau, H., Delmaire, C., Capelle, L., Gatignol, P., Ducros, M., Chiras, J., \& Lehéricy, S. (2010). Comparison of diffusion tensor imaging tractography of language tracts and intraoperative subcortical stimulations. Journal of Neurosurgery, 112(3), 503-511.

Lee, D. D., \& Seung, H. S. (1999). Learning the parts of objects by non-negative matrix factorization. Nature, 401(6755), 788-791.

Lee, D. W., Lee, J. Y., Ryu, S. G., Cho, S. J., Hong, C. H., Lee, J. H., Choi, Y. M., Kim, B. S., Park, E. J., \& Park, S. H. (2005). Validity of the Korean version of Informant Questionnaire on the Cognitive Decline in the Elderly (IQCODE). Journal of the Korean Geriatrics Society, 9(3), 196-202.

Levy, J. (1978). Lateral differences in the human brain in cognition and behavioral control. Cerebral Correlates of Conscious Experience, 1, 202-208.

Luengo-Fernandez, R., Violato, M., Candio, P., \& Leal, J. (2020). Economic burden of stroke across Europe: A population-based cost analysis. European Stroke Journal, 5(1), 17-25.

Lunven, M., Thiebaut De Schotten, M., Bourlon, C., Duret, C., Migliaccio, R., Rode, G., \& Bartolomeo, P. (2015). White matter lesional predictors of chronic visual neglect: a longitudinal study. Brain: A Journal of Neurology, 138(Pt 3), 746-760.

Marchina, S., Zhu, L. L., Norton, A., Zipse, L., Wan, C. Y., \& Schlaug, G. (2011). Impairment of speech production predicted by lesion load of the left arcuate fasciculus. Stroke; a Journal of Cerebral Circulation, 42(8), 2251-2256.

Marcotte, K., Perlbarg, V., Marrelec, G., Benali, H., \& Ansaldo, A. I. (2013). Default-mode network functional connectivity in aphasia: therapy-induced neuroplasticity. Brain and Language, 124(1), 45-55.

Margulies, D. S., Ghosh, S. S., Goulas, A., Falkiewicz, M., Huntenburg, J. M., Langs, G., Bezgin, G., Eickhoff, S. B., Castellanos, F. X., Petrides, M., Jefferies, E., \& Smallwood, J. (2016). Situating the default-mode network along a principal gradient of macroscale cortical organization. Proceedings of the National Academy of Sciences of the United States of America, 113(44), 12574-12579.

Mirman, D., Chen, Q., Zhang, Y., Wang, Z., Faseyitan, O. K., Coslett, H. B., \& Schwartz, M. F. (2015). Neural 
organization of spoken language revealed by lesion-symptom mapping. Nature Communications, 6(1), $1-9$.

Musso, M., Weiller, C., Kiebel, S., Müller, S. P., Bülau, P., \& Rijntjes, M. (1999). Training-induced brain plasticity in aphasia. Brain: A Journal of Neurology, 122 ( Pt 9), 1781-1790.

Nord, C. L., Valton, V., Wood, J., \& Roiser, J. P. (2017). Power-up: a reanalysis of'power failure'in neuroscience using mixture modeling. Journal of Neuroscience, 37(34), 8051-8061.

Olesen, J., Gustavsson, A., Svensson, M., Wittchen, H.-U., Jönsson, B., CDBE2010 study group, \& European Brain Council. (2012). The economic cost of brain disorders in Europe. European Journal of Neurology: The Official Journal of the European Federation of Neurological Societies, 19(1), 155-162.

Papagno, C., Miracapillo, C., Casarotti, A., Romero Lauro, L. J., Castellano, A., Falini, A., Casaceli, G., Fava, E., \& Bello, L. (2011). What is the role of the uncinate fasciculus? Surgical removal and proper name retrieval. Brain: A Journal of Neurology, 134(Pt 2), 405-414.

Parker, G. J. M., Luzzi, S., Alexander, D. C., Wheeler-Kingshott, C. A. M., Ciccarelli, O., \& Lambon Ralph, M. A. (2005). Lateralization of ventral and dorsal auditory-language pathways in the human brain. NeuroImage, 24(3), 656-666.

Pedersen, P., Vinter, K., \& Olsen, T. S. (2004). Aphasia after Stroke: Type, Severity and Prognosis. Cerebrovascular Diseases , 17(1), 35-43.

Pobric, G., Jefferies, E., \& Ralph, M. A. L. (2007). Anterior temporal lobes mediate semantic representation: mimicking semantic dementia by using rTMS in normal participants. Proceedings of the National Academy of Sciences of the United States of America, 104(50), 20137-20141.

Pustina, D., Avants, B., Faseyitan, O. K., Medaglia, J. D., \& Coslett, H. B. (2018). Improved accuracy of lesion to symptom mapping with multivariate sparse canonical correlations. Neuropsychologia, 115, 154-166.

Rauschecker, J. P., \& Tian, B. (2000). Mechanisms and streams for processing of "what" and "where" in auditory cortex. Proceedings of the National. https://www.pnas.org/content/97/22/11800.short

Reeves, M. J., Bushnell, C. D., Howard, G., Gargano, J. W., Duncan, P. W., Lynch, G., Khatiwoda, A., \& Lisabeth, L. (2008). Sex differences in stroke: epidemiology, clinical presentation, medical care, and outcomes. Lancet Neurology, 7(10), 915-926.

Rilling, J. K., Glasser, M. F., Preuss, T. M., Ma, X., Zhao, T., Hu, X., \& Behrens, T. E. J. (2008). The evolution of the arcuate fasciculus revealed with comparative DTI. Nature Neuroscience, 11(4), 426-428.

Ritchie, S. J., Cox, S. R., Shen, X., Lombardo, M. V., Reus, L. M., Alloza, C., Harris, M. A., Alderson, H. L., Hunter, S., Neilson, E., Liewald, D. C. M., Auyeung, B., Whalley, H. C., Lawrie, S. M., Gale, C. R., Bastin, M. E., McIntosh, A. M., \& Deary, I. J. (2018). Sex Differences in the Adult Human Brain: Evidence from 5216 UK Biobank Participants. Cerebral Cortex, 28(8), 2959-2975.

Ritter, F., Boskamp, T., Homeyer, A., Laue, H., Schwier, M., Link, F., \& Peitgen, H.-O. (2011). Medical image analysis. IEEE Pulse, 2(6), 60-70.

Ruigrok, A. N. V., Salimi-Khorshidi, G., Lai, M.-C., Baron-Cohen, S., Lombardo, M. V., Tait, R. J., \& Suckling, J. (2014). A meta-analysis of sex differences in human brain structure. Neuroscience and Biobehavioral Reviews, 39, 34-50.

Salvatier, J., Wiecki, T. V., \& Fonnesbeck, C. (2016). Probabilistic programming in Python using PyMC3. In PeerJ Computer Science (Vol. 2, p. e55). https://doi.org/10.7717/peerj-cs.55

Saur, D., Kreher, B. W., Schnell, S., Kümmerer, D., Kellmeyer, P., Vry, M.-S., Umarova, R., Musso, M., Glauche, V., Abel, S., Huber, W., Rijntjes, M., Hennig, J., \& Weiller, C. (2008). Ventral and dorsal pathways for language. Proceedings of the National Academy of Sciences of the United States of America, 105(46), 18035-18040.

Schmahmann, J. D., Doyon, J., Petrides, M., Evans, A. C., \& Toga, A. W. (2000). MRI Atlas of the Human Cerebellum. Academic Press.

Shaywitz, B. A., Shaywitz, S. E., Pugh, K. R., Constable, R. T., Skudlarski, P., Fulbright, R. K., Bronen, R. A., Fletcher, J. M., Shankweiler, D. P., \& Katz, L. (1995). Sex differences in the functional organization of the brain for language. Nature, 373(6515), 607-609.

Siegel, J. S., Ramsey, L. E., Snyder, A. Z., Metcalf, N. V., Chacko, R. V., Weinberger, K., Baldassarre, A., Hacker, C. D., Shulman, G. L., \& Corbetta, M. (2016). Disruptions of network connectivity predict impairment in multiple behavioral domains after stroke. Proceedings of the National Academy of Sciences 
of the United States of America, 113(30), E4367-E4376.

Silva, G. S., Lima, F. O., Camargo, E. C. S., Smith, W. S., Lev, M. H., Harris, G. J., Halpern, E. F., Koroshetz, W., \& Furie, K. L. (2010). Gender differences in outcomes after ischemic stroke: role of ischemic lesion volume and intracranial large-artery occlusion. Cerebrovascular Diseases , 30(5), 470-475.

Smith, D. V., Clithero, J. A., Rorden, C., \& Karnath, H.-O. (2013). Decoding the anatomical network of spatial attention. Proceedings of the National Academy of Sciences of the United States of America, 110(4), $1518-1523$.

Sperber, C. (2020). Rethinking causality and data complexity in brain lesion-behaviour inference and its implications for lesion-behaviour modelling. Cortex; a Journal Devoted to the Study of the Nervous System and Behavior, 126, 49-62.

Stockert, A., Wawrzyniak, M., Klingbeil, J., Wrede, K., Kümmerer, D., Hartwigsen, G., Kaller, C. P., Weiller, C., \& Saur, D. (2020). Dynamics of language reorganization after left temporo-parietal and frontal stroke. Brain: A Journal of Neurology, 143(3), 844-861.

Thiebaut de Schotten, M., Ffytche, D. H., Bizzi, A., Dell'Acqua, F., Allin, M., Walshe, M., Murray, R., Williams, S. C., Murphy, D. G. M., \& Catani, M. (2011). Atlasing location, asymmetry and inter-subject variability of white matter tracts in the human brain with MR diffusion tractography. NeuroImage, 54(1), 49-59.

Thomas Yeo, B. T., Krienen, F. M., Sepulcre, J., Sabuncu, M. R., Lashkari, D., Hollinshead, M., Roffman, J. L., Smoller, J. W., Zöllei, L., Polimeni, J. R., Fischl, B., Liu, H., \& Buckner, R. L. (2011). The organization of the human cerebral cortex estimated by intrinsic functional connectivity. Journal of Neurophysiology, 106(3), 1125-1165.

Tuladhar, A. M., Snaphaan, L., Shumskaya, E., Rijpkema, M., Fernandez, G., Norris, D. G., \& de Leeuw, F.-E. (2013). Default Mode Network Connectivity in Stroke Patients. PloS One, 8(6), e66556.

Ueno, T., Saito, S., Rogers, T. T., \& Lambon Ralph, M. A. (2011). Lichtheim 2: synthesizing aphasia and the neural basis of language in a neurocomputational model of the dual dorsal-ventral language pathways. Neuron, 72(2), 385-396.

Ullman, M. T., Miranda, R. A., \& Travers, M. L. (2007). Sex differences in the neurocognition of language. In Sex Differences in the Brain (pp. 291-310). Oxford University Press.

Vigneau, M., Beaucousin, V., Hervé, P. Y., Duffau, H., Crivello, F., Houdé, O., Mazoyer, B., \& Tzourio-Mazoyer, N. (2006). Meta-analyzing left hemisphere language areas: phonology, semantics, and sentence processing. NeuroImage, 30(4), 1414-1432.

Wafa, H. A., Wolfe, C. D. A., Emmett, E., Roth, G. A., Johnson, C. O., \& Wang, Y. (2020). Burden of Stroke in Europe: Thirty-Year Projections of Incidence, Prevalence, Deaths, and Disability-Adjusted Life Years. Stroke; a Journal of Cerebral Circulation, 51(8), 2418-2427.

Wallentin, M. (2009). Putative sex differences in verbal abilities and language cortex: a critical review. Brain and Language, 108(3), 175-183.

Wang, C., Qin, W., Zhang, J., Tian, T., Li, Y., Meng, L., Zhang, X., \& Yu, C. (2014). Altered functional organization within and between resting-state networks in chronic subcortical infarction. Journal of Cerebral Blood Flow and Metabolism: Official Journal of the International Society of Cerebral Blood Flow and Metabolism, 34(4), 597-605.

Wang, J., Marchina, S., Norton, A. C., Wan, C. Y., \& Schlaug, G. (2013). Predicting speech fluency and naming abilities in aphasic patients. Frontiers in Human Neuroscience, 7, 831.

Weaver, N. A., Zhao, L., Biesbroek, J. M., Kuijf, H. J., Aben, H. P., Bae, H.-J., Caballero, M. Á. A., Chappell, F. M., Chen, C. P. L. H., Dichgans, M., Duering, M., Georgakis, M. K., van der Giessen, R. S., Gyanwali, B., Hamilton, O. K. L., Hilal, S., Vom Hofe, E. M., de Kort, P. L. M., Koudstaal, P. J., ... Biessels, G. J. (2019). The Meta VCI Map consortium for meta-analyses on strategic lesion locations for vascular cognitive impairment using lesion-symptom mapping: Design and multicenter pilot study. Alzheimer's \& Dementia: The Journal of the Alzheimer's Association, 11(1), 310-326.

Woollams, A. M. (2012). Apples are not the only fruit: the effects of concept typicality on semantic representation in the anterior temporal lobe. Frontiers in Human Neuroscience, 6, 85.

Wu, O., Cloonan, L., Mocking, S. J. T., Bouts, M. J. R. J., Copen, W. A., Cougo-Pinto, P. T., Fitzpatrick, K., Kanakis, A., Schaefer, P. W., Rosand, J., Furie, K. L., \& Rost, N. S. (2015). Role of Acute Lesion 
Topography in Initial Ischemic Stroke Severity and Long-Term Functional Outcomes. Stroke; a Journal of Cerebral Circulation, 46(9), 2438-2444.

Xu, M., Liang, X., Ou, J., Li, H., Luo, Y.-J., \& Tan, L. H. (2020). Sex Differences in Functional Brain Networks for Language. Cerebral Cortex, 30(3), 1528-1537.

Yu, K.-H., Cho, S.-J., Oh, M. S., Jung, S., Lee, J.-H., Shin, J.-H., Koh, I.-S., Cha, J.-K., Park, J.-M., Bae, H.-J., Kang, Y., Lee, B.-C., \& Korean-Vascular Cognitive Impairment Harmonization Standards Study Group. (2013). Cognitive impairment evaluated with Vascular Cognitive Impairment Harmonization Standards in a multicenter prospective stroke cohort in Korea. Stroke; a Journal of Cerebral Circulation, 44(3), 786-788.

Yu, Q., Wang, H., Li, S., \& Dai, Y. (2019). Predictive role of subcomponents of the left arcuate fasciculus in prognosis of aphasia after stroke: A retrospective observational study. Medicine, 98(23), e15775.

Zeestraten, E. A., Gudbrandsen, M. C., Daly, E., de Schotten, M. T., Catani, M., Dell'Acqua, F., Lai, M.-C., Ruigrok, A. N. V., Lombardo, M. V., Chakrabarti, B., Baron-Cohen, S., Ecker, C., MRC AIMS Consortium, Murphy, D. G. M., \& Craig, M. C. (2017). Sex differences in frontal lobe connectivity in adults with autism spectrum conditions. Translational Psychiatry, 7(4), e1090.

Zhang, J., Wei, X., Xie, S., Zhou, Z., Shang, D., Ji, R., Yu, Y., He, F., Du, Y., Ye, X., \& Luo, B. (2018). Multifunctional Roles of the Ventral Stream in Language Models: Advanced Segmental Quantification in Post-Stroke Aphasic Patients. Frontiers in Neurology, 9, 89.

Zhang, Y., Wang, L., Yang, J., Yan, R., Zhang, J., Sang, L., Li, P., Liu, H., \& Qiu, M. (2017). Abnormal functional networks in resting-state of the sub-cortical chronic stroke patients with hemiplegia. Brain Research, 1663, 51-58.

Zhao, Z., Wu, J., Fan, M., Yin, D., Tang, C., Gong, J., Xu, G., Gao, X., Yu, Q., Yang, H., Sun, L., \& Jia, J. (2018). Altered intra- and inter-network functional coupling of resting-state networks associated with motor dysfunction in stroke. Human Brain Mapping, 39(8), 3388-3397. 


\section{Figures}
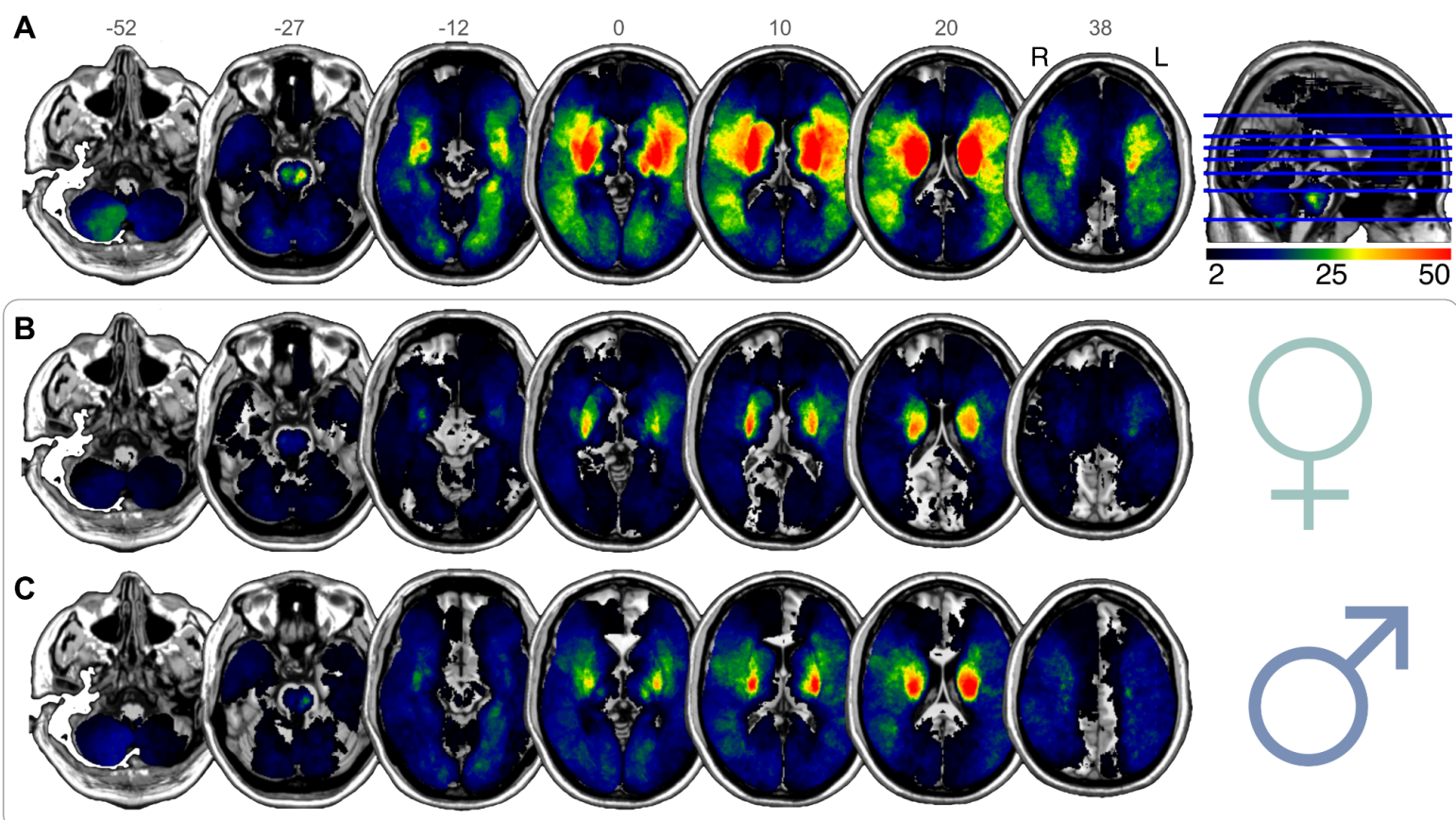

Figure 1. Spatial topography of ischemic stroke lesions summarized from 1,401 patients.

We confirm excellent whole-brain coverage, without appreciable hemispheric differences in the voxel-level stroke topography for the whole cohort (A), or separately either for female patients $(\mathbf{B})$ or for male patients $(\mathbf{C})$. The overall lesion volume did not significantly differ between female and male patients within our cohort (two-sided t-test, $p=0.13$, cf. Table 1 ). The topographical distributions of tissue damage caused by ischemic stroke are shown in MNI reference space with z-coordinates indicated above each brain slice. Lesions predominantly overlapped in areas that correspond to the vascular territory of the left and right middle cerebral artery. The majority of lesions were localized in subcortical zones, which entails a disconnection of deep white matter tracts. Radiological view; R/L: right/left. 
A

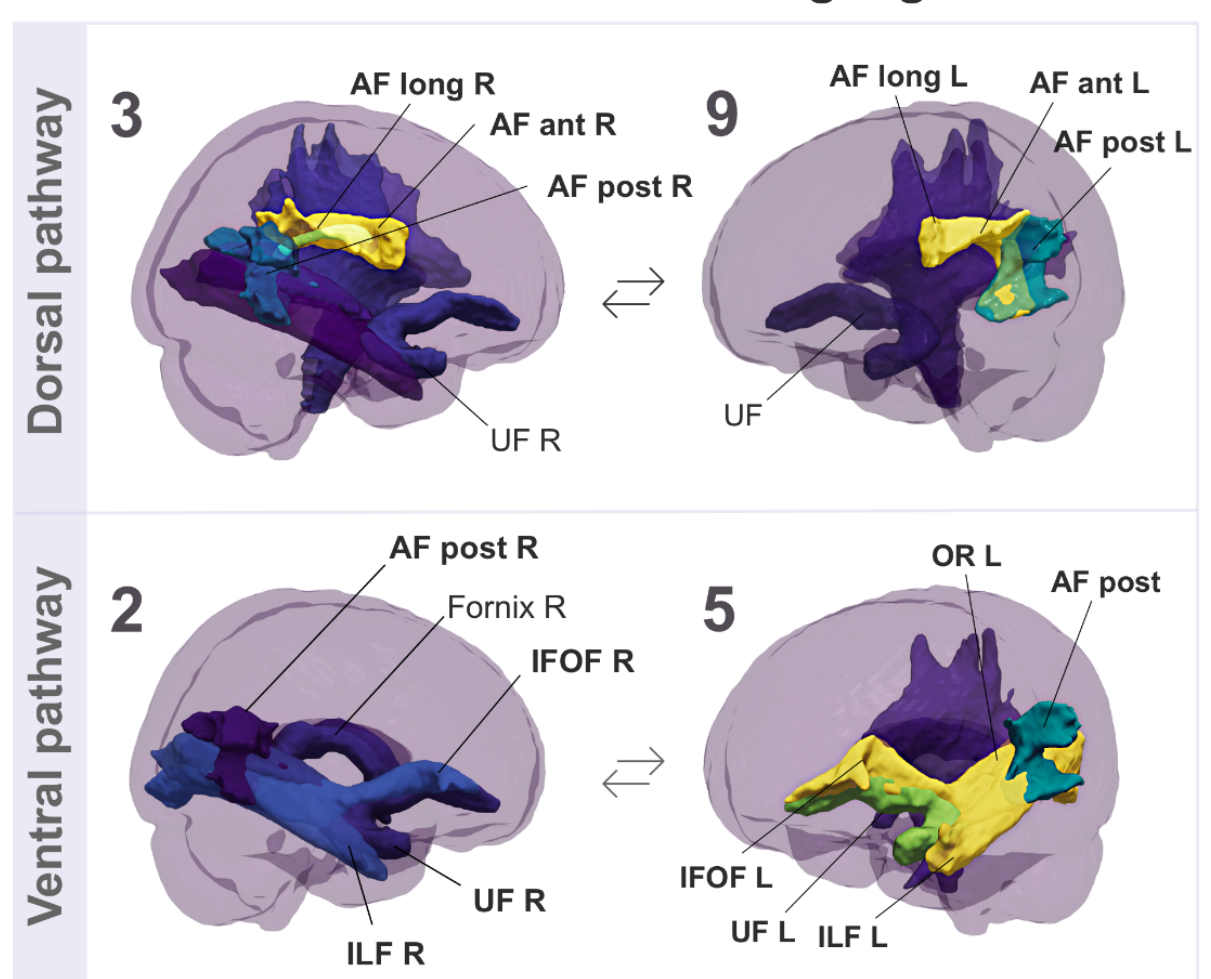

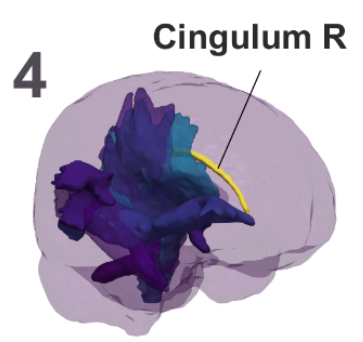

Cingulum L

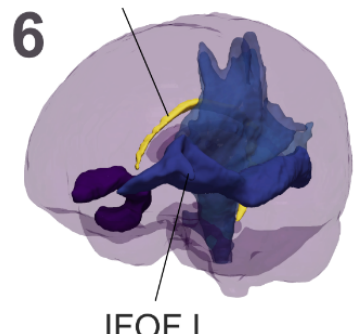

IFOF L

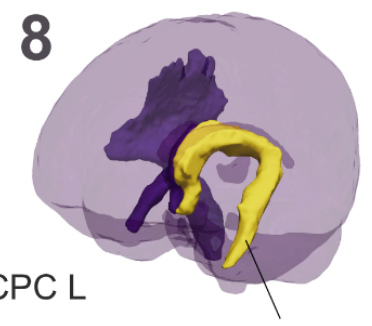

Fornix L

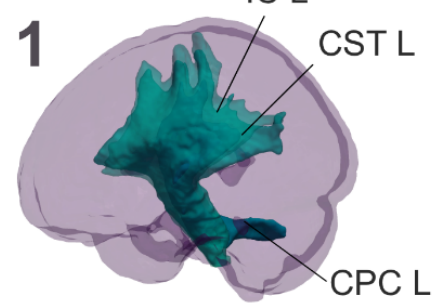

IC R
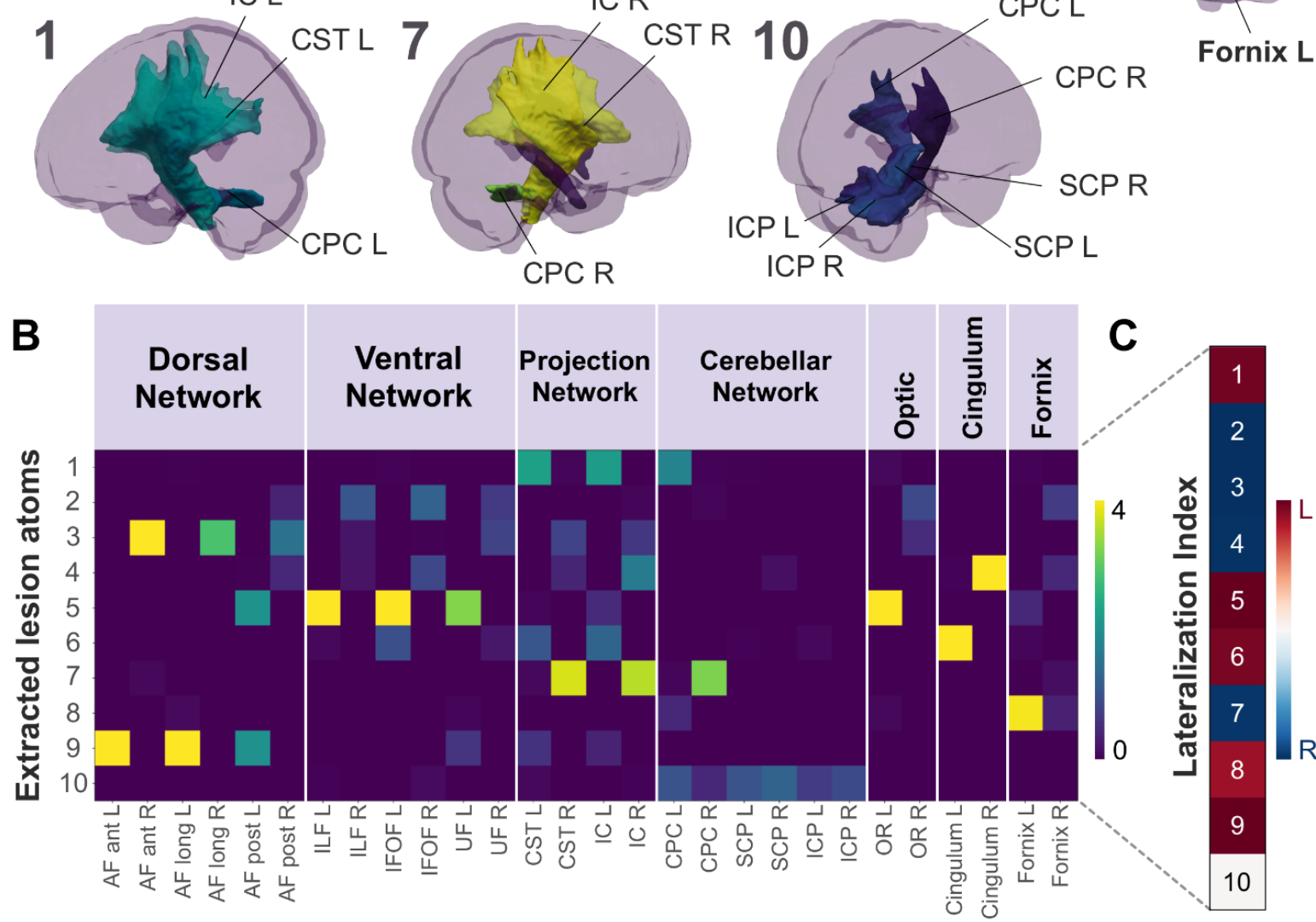


\section{Figure 2. Multivariate latent-factor discovery deconvolves unique stroke lesion atoms.}

In 1,401 neurological patients, we used an unsupervised machine learning algorithm to perform a data-driven discovery of white matter lesion patterns. This sum-of-parts approach (non-negative matrix factorization, NNMF) enabled the identification of archetypical white-matter lesion configurations, lesion atoms, distilled from high-resolution brain scans with a total $>1,7$ million $1 \mathrm{~mm}^{3}$ lesion voxels across patients. The anatomical distribution of the derived lesion atoms corresponded to directly interpretable and biologically plausible lesion patterns that are reminiscent of well-known functional systems (B). Each atom of coherent white matter lesion was assessed for hemispheric asymmetry (C) based on a lateralization index (LI) (Ito \& Liew, 2016). This index is computed from the weights of the latent factor loadings by $L I=(L-R) /(L+R)$. Hemispheric asymmetry effects are indicated by left- (Red) or right-lateralization (Blue). Most factors showed a left-hemispheric lesion configuration in correspondence with a similar right-hemispheric homolog. Four factors were predominantly associated with the language-related pathways (A, Box): the dorsal perisylvian circuitry (Factor 3 and 9; R and L, respectively) and the ventral pathway (Factor 2 and 5; R and L, respectively). Notably, the core perisylvian pathway shows significant leftward asymmetry in factor 9 with the accentuated influence of the long and anterior AF. Instead, the right homolog in factor 3 predominantly relies on the anterior segment of the AF. Motor function (A, bottom row) was most strongly expressed in factors 1 (L) and 7 (R), and 10 (bilateral cerebellar network). Primarily limbic factors 4 and 6 were dominated by the influence of the left and right cingulum. Factor 8 was uniquely left-lateralized and captured the main effect of the fornix (A, right column). As such, even subtle differences in lateralization align with the current neuroanatomical knowledge of the language pathways (Thiebaut de Schotten et al., 2011).

Abbreviations: Arcuate fasciculus: AF. Inferior longitudinal fasciculus: ILF. Uncinate fasciculus: UF. Inferior fronto-occipital fasciculus: IFOF. Cortico-spinal tract: CST. Internal capsule: IC. Cortico-pontine-cerebellar tract: CPC. Inferior and superior cerebellar peduncle: ICP, SCP. Optic radiation: OR. R/L: right/left. 


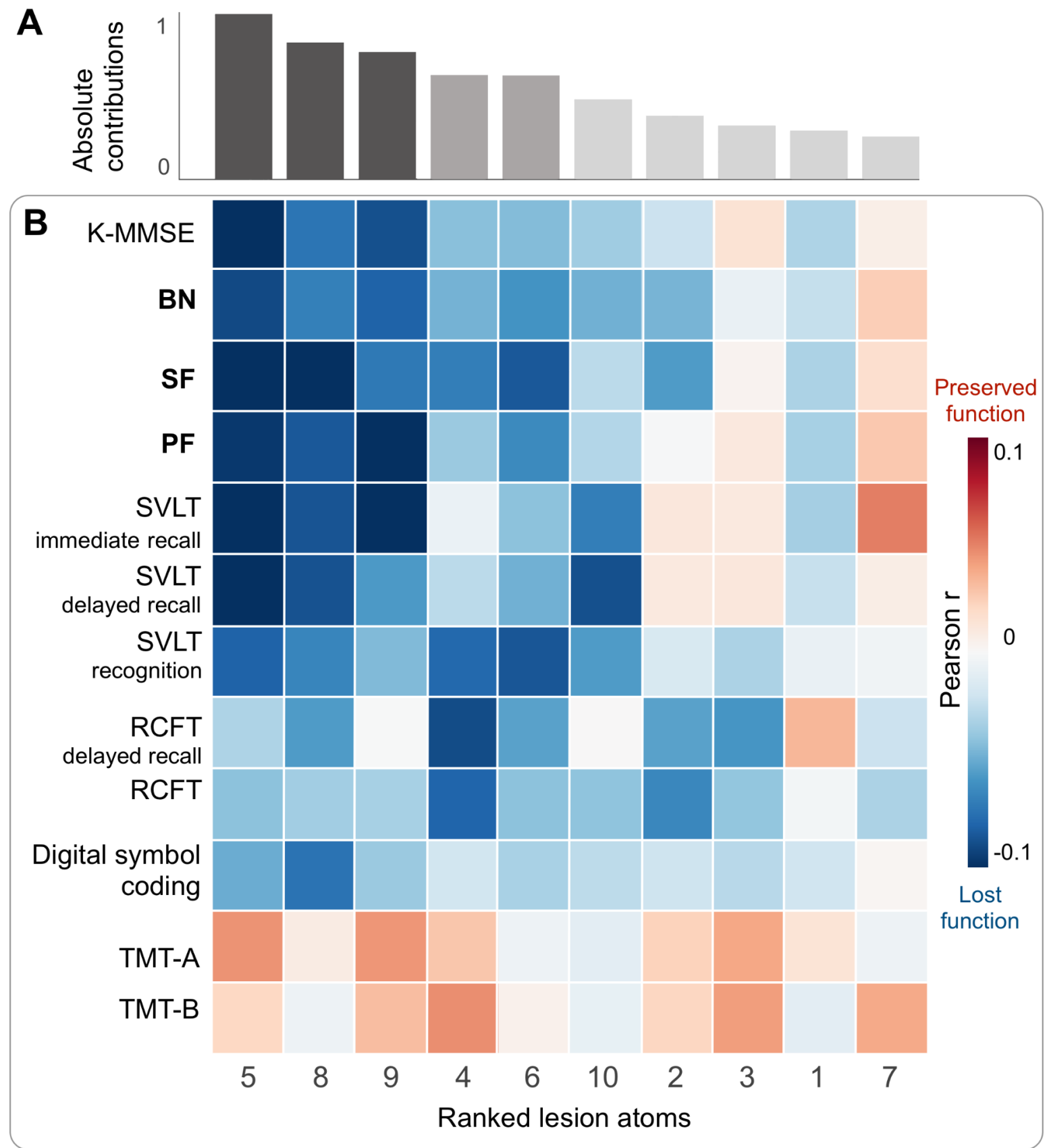

Figure 3. Stroke lesion atoms are ranked from strongest to weakest associations with clinical outcomes.

We assessed the overall clinical relevance of each lesion atom using Pearson's correlation between subject-specific lesion atom expression and a cognitive test across outcome measures. A) Based on the summed absolute contributions, factors 5 (Ventral pathway L), 8 (Fornix L), and 9 (Dorsal pathway L) ranked as the three most relevant lesion atoms. Following the top three lesion atoms, the limbic factors 4 and 6 reflect the right and left influence of the cingular fiber bundles. B) All top five lesion atoms showed pronounced negative associations with cognitive impairment across most 
neuropsychological assessments. Regarding language, damage in the left-hemispheric lesion atoms (Factors 5 and 9) of the ventral and dorsal pathway cause a significant degree of deterioration in the Boston naming test (BN), semantic and phonemic fluency (SF, PF). However, the association with their respective right-hemispheric homologs diverged: the right-hemispheric homolog of the dorsal core perisylvian network (Factor 3) showed slight negative correlation in BN, but otherwise positive correlations in SF and PF. In contrast, the right-hemispheric homolog of the ventral pathway (Factor 2 ) is associated with a considerable deterioration in $\mathrm{BN}$ and $\mathrm{SF}$.

Abbreviations: Korean-Mini Mental State Examination: K-MMSE. Boston Naming: BN. Semantic fluency: SF. Phonemic fluency: PF. Seoul-Verbal Learning: SVL. Rey Complex Figure Test: RCFT. Korean-Trail Making Test Version A/B: TMT A/B. R: right, L: left. 

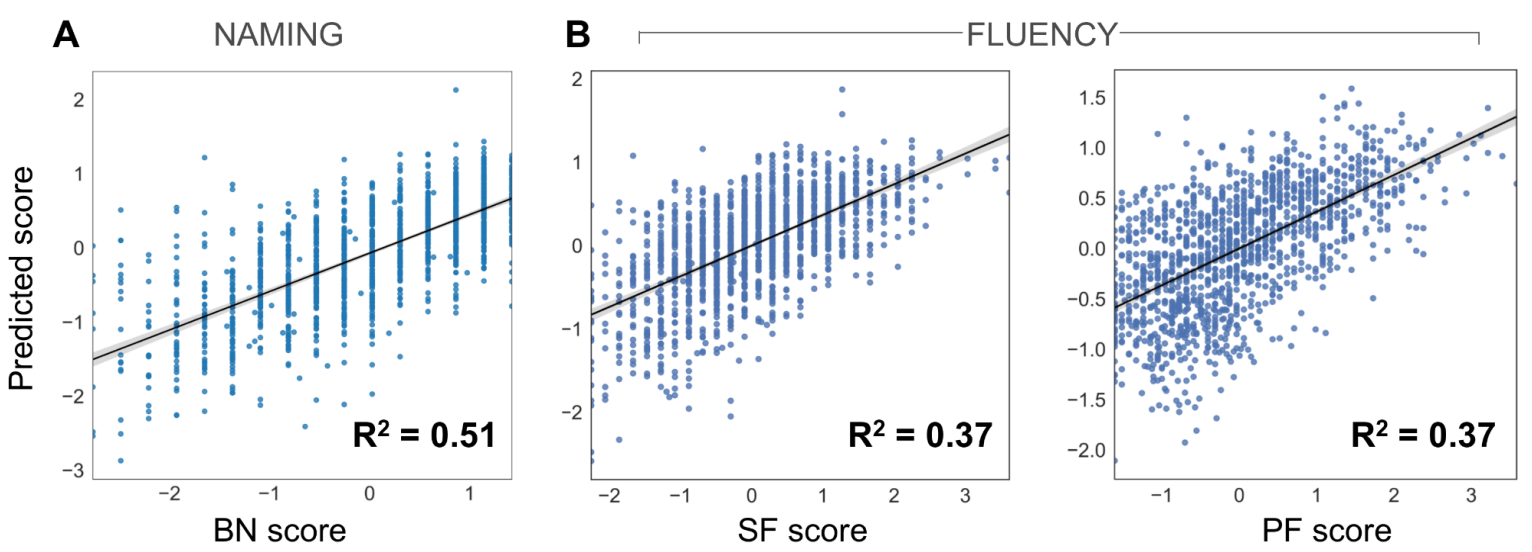

\section{Figure 4. Performance of the inferred Bayesian analytical solution in predicting naming and speech fluency.}

We confirmed a well-fitted approximation of the underlying distribution as performed for model checking in previous research (Bonkhoff, Lim, Bae, Weaver, Kuijf, et al., 2021; Kiesow et al., 2021). The posterior predictive checks are shown for the Bayesian models that were estimated to predict inter-individual difference in (z-scored) Boston naming ( $\mathbf{A}, \mathrm{BN})$, semantic and phonemic speech fluency (B, SF and PF). These model-based simulations of new data were then compared to the actually observed data to compute the overall explained variance (coefficient of determination, $\mathrm{R}^{2}$ ). This practical check of model-based outcome predictions is a well-recognized approximation to external validation given the actual data at hand (Kruschke, 2014). The full model for the BN outcome explained a total variance of $\mathrm{R}^{2}=0.51$, and the Bayesian models for the $P F$ and $S F$ explained $\mathrm{R}^{2}=0.37$, respectively. Considering our white matter results, the predictive performance for naming and speech fluency matches previous research on grey matter lesions due to stroke (Bonkhoff, Lim, Bae, Weaver, Kuijf, et al., 2021). 


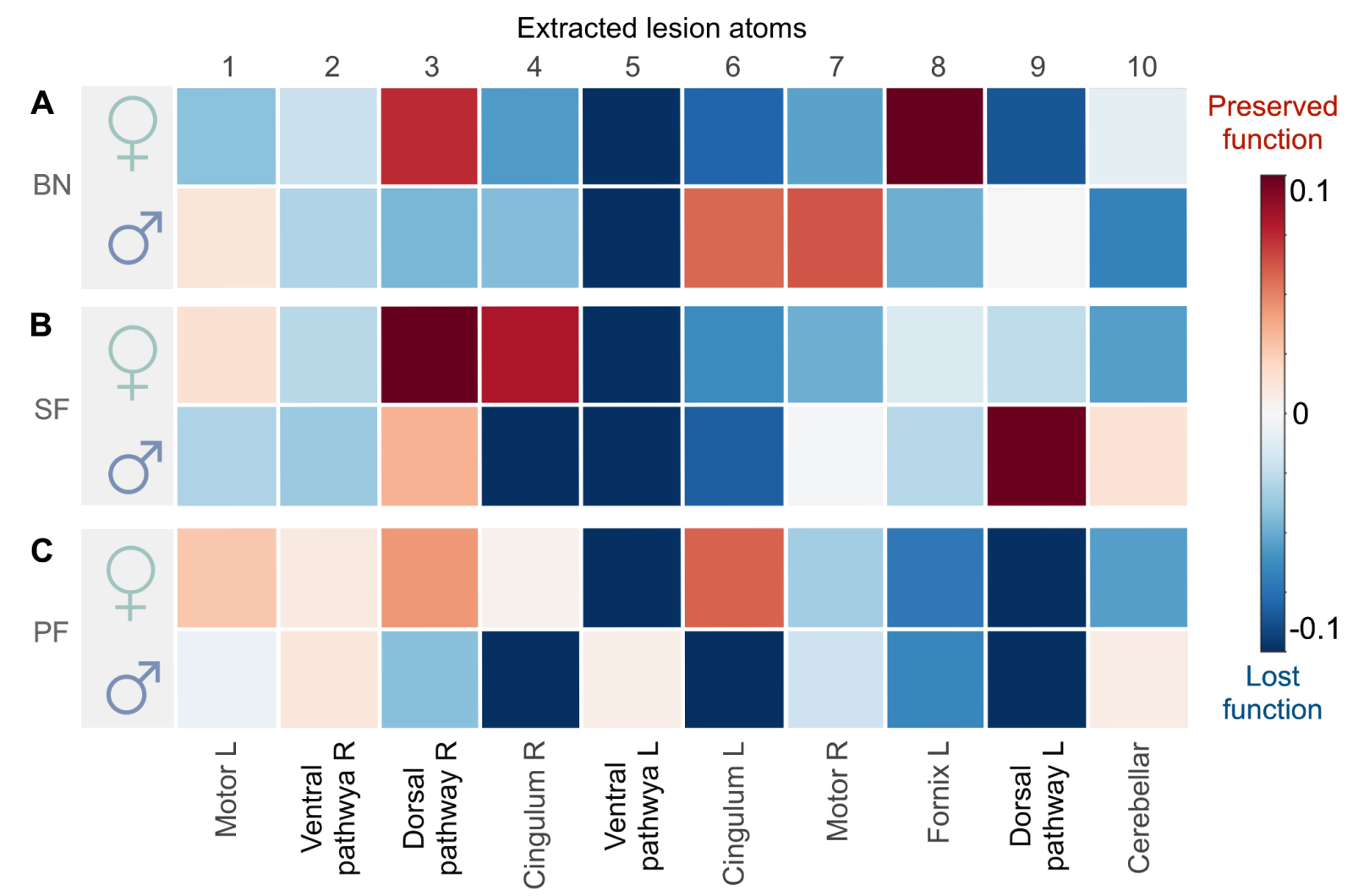

Figure 5. Sex bias becomes apparent from contributions of specific lesion atoms to explaining language outcomes.

For each lesion atom, we show the predictive role in explaining language function separately for women and men. Shows the mean marginal Baysian posterior distribution of each lesion atom to explain moderate to severe language impairment. Across sexes, effects can be similar or diverging. A) For the Boston naming (BN), we observed driving loss of function common to both sexes in the left-lateralized ventral pathway (Factor 5), its right-hemispheric homolog (Factor 2), and the right cingulum (Factor 4). Diverging lesion effects between females and males arise in several atoms, including the right dorsal pathway, the left fornix, cingular tract, and the left and right motor projections (Factors 3, 8, 6, 7 and 1 respectively). B) Similarly for semantic fluency (SF), the main effect common to both sexes leading to worse performance was in the left ventral pathway (Factor 5) as well. Less influential were common effects in the right ventral pathway, the left cingulum and fornix (Factors 2, 6, 8). Analogous diverging lesion-outcome predictions in the right dorsal perisylvian circuit (Factor 3) were less pronounced than in BN, leading to moderate impairment for both sexes. But lesions in the left dorsal pathway lead to worse outcomes specifically for females. We further observed differences between women and men in the lesioned right cingulum (Factor 4). Here, males were predicted to have a more severe impairment. C) For phonemic fluency (PF), adverse outcome is primarily explained by the contributions of the left dorsal pathway (Factor 9) and left Fornix (Factor 8) in both sexes. We observed marked differences between sexes for the 
bioRxiv preprint doi: https://doi.org/10.1101/2022.01.18.474989; this version posted January $18,2022$. The copyright holder for this preprint (which was not certified by peer review) is the author/funder, who has granted bioRxiv a license to display the preprint in perpetuity. It is made available under aCC-BY-NC-ND 4.0 International license.

right-hemispheric dorsal pathway, left and right cingulum, with males performing worse than females (Factor 3, 4 and 6). In contrast, women showed significantly worse outcomes in the lesioned left ventral pathway (Factor 5). 


\section{Shared effects}
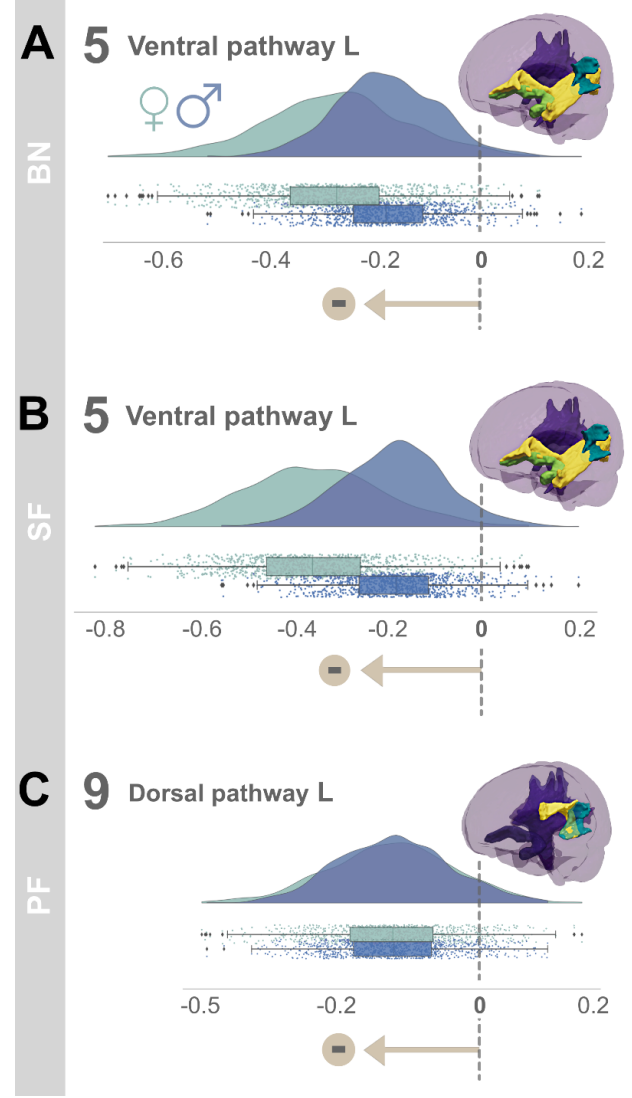

\section{Diverging effects}
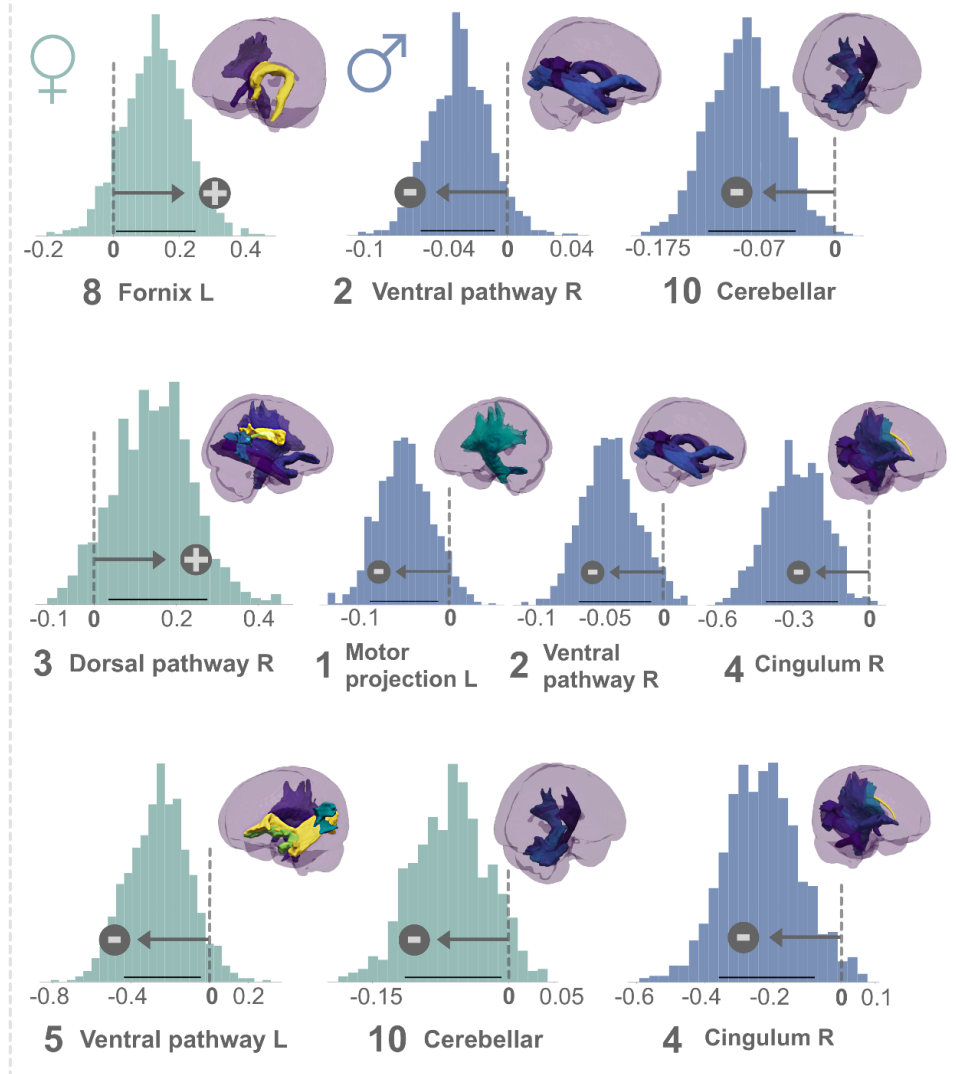

Figure 6. Shared and diverging lesion effects on language performance outcomes in female and male stroke populations.

We formed full posterior-Bayes estimates to infer the explanatory relevance of each lesion atom for the prediction of language outcomes in female and male stroke patients. Key assessments of post-stroke language performance included the Boston Naming test (BN, A), semantic (SF, B) and phonemic fluency $(\mathrm{PF}, \mathbf{C})$. Lesion atoms were considered relevant if the highest probability density interval (HPDI) of the posterior distribution covering 80\% certainty (black line) did not overlap with zero. We found lesion pattern effects that were common to both sexes (left side) and that diverged between women and men (right side). Across both sexes, lesion topographies pointed to an overarching structural organization reflecting the dual-stream concept of language (Gregory Hickok \& Poeppel, 2004). Here, the ventral pathway was critically involved in naming and semantic fluency deficits, and the dorsal pathway in phonemic fluency deficits, congruently for women and men. More importantly, we provide novel evidence for sex-nuanced lesion-outcome effects. Women primarily rely on left-dominant circuits, including structural connections of the fornix, and a female-specific vulnerability to ventral lesion damage in phonemic fluency. In contrast, men draw from bilateral 
bioRxiv preprint doi: https://doi.org/10.1101/2022.01.18.474989; this version posted January $18,2022$. The copyright holder for this preprint (which was not certified by peer review) is the author/funder, who has granted bioRxiv a license to display the preprint in perpetuity. It is made available under aCC-BY-NC-ND 4.0 International license.

ventral and cerebellar connections, and potentially integrate more domain-general cognitive and executive functions via right-lateralized cingular fiber bundle to enable efficient language processing after stroke. 


\section{Tables}

\section{Table 1. Characteristics of the patient cohort.}

Variables depicted as mean (standard deviation). Sex differences were tested using independent t-test ( $t, p$-value), or in case of unequal variances using Welch's t-test ( $t, p$-value).

\begin{tabular}{|c|c|c|c|c|}
\hline & Cohort & Women & Men & Sex differences \\
\hline Age & $67.58(11.59)$ & $70.73(11.15)$ & $65.29(11.38)$ & $8.9,<0.001$ \\
\hline Lesion volume & $17.08(43.33)$ & $15.05(44.16)$ & $18.57(42.70)$ & $-1.5,0.1337$ \\
\hline IQCODE & $3.36(0.46)$ & $3.42(0.49)$ & $3.32(0.44)$ & $3.91,<0.001$ \\
\hline Years of education & $9.28(5.20)$ & $6.68(4.98)$ & $11.16(4.51)$ & $-17.27,<0.001$ \\
\hline K-MMSE & $23.73(5.92)$ & $21.93(6.34)$ & $25.03(5.23)$ & $-9.98,<0.001$ \\
\hline Boston naming (BN) & $9.92(3.59)$ & $8.63(3.54)$ & $10.86(3.32)$ & $-11.90,<0.001$ \\
\hline Semantic fluency (SF) & $11.51(5.11)$ & $10.39(4.89)$ & $12.33(5.12)$ & $-7.12,<0.001$ \\
\hline Phonemic fluency (PF) & $17.37(10.78)$ & $14.76(10.16)$ & $19.27(10.82)$ & $-7.97,<0.001$ \\
\hline SVLT immediate recall & $14.82(6.01)$ & $14.71(6.45)$ & $14.90(5.67)$ & $-0.57,0.5$ \\
\hline SVLT delayed recall & $3.67(2.92)$ & $3.73(3.04)$ & $3.64(2.82)$ & $0.54,0.5$ \\
\hline SVLT recognition & $18.16(4.14)$ & $17.96(4.14)$ & $18.31(4.14)$ & $-1.55,0.1$ \\
\hline RCFT delayed recall & $11.33(5.83)$ & $9.49(5.38)$ & $12.66(5.78)$ & $-10.42,<0.001$ \\
\hline RCFT & $25.74(8.68)$ & $23.09(9.35)$ & $27.67(7.60)$ & $-10.09,<0.001$ \\
\hline Digital symbol coding & $35.67(21.73)$ & $29.35(20.90)$ & $40.26(21.17)$ & $-9.56,<0.001$ \\
\hline TMT-A & $42.53(36.52)$ & $52.81(46.70)$ & $35.11(24.36)$ & $9.21,<0.001$ \\
\hline TMT-B & $95.58(76.56)$ & $117.80(85.56)$ & $79.28(64.41)$ & $9.61,<0.001$ \\
\hline
\end{tabular}




\title{
Supplementary Online Material
}

K-MMSE
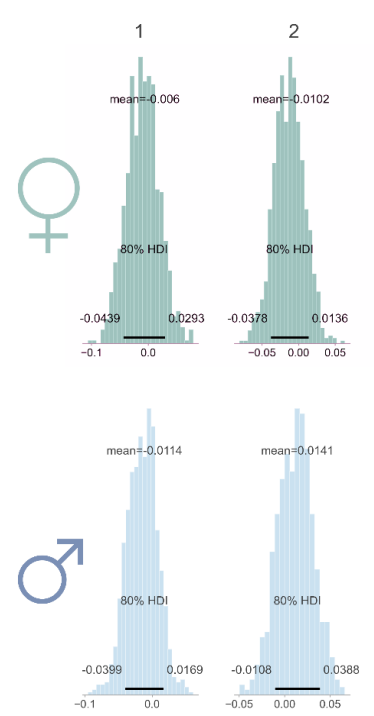
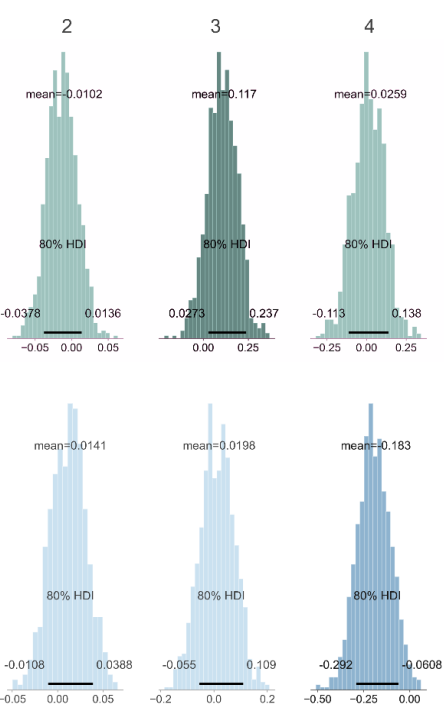
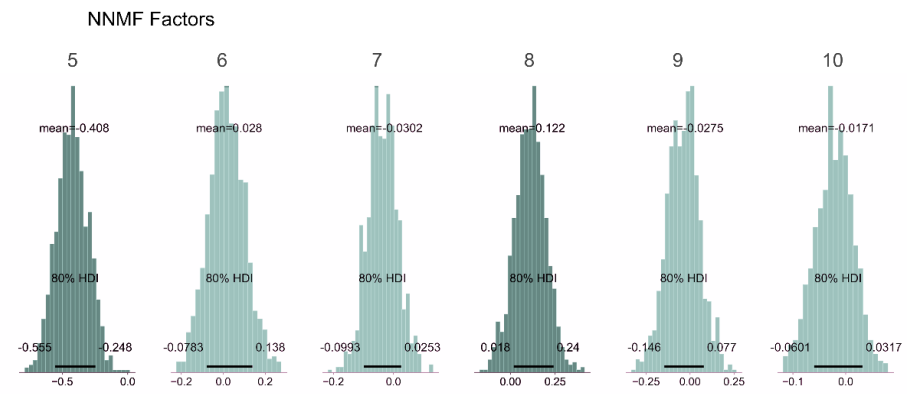

\section{Supp. Figure 1. K-MMSE Bayesian posteriors}

BN
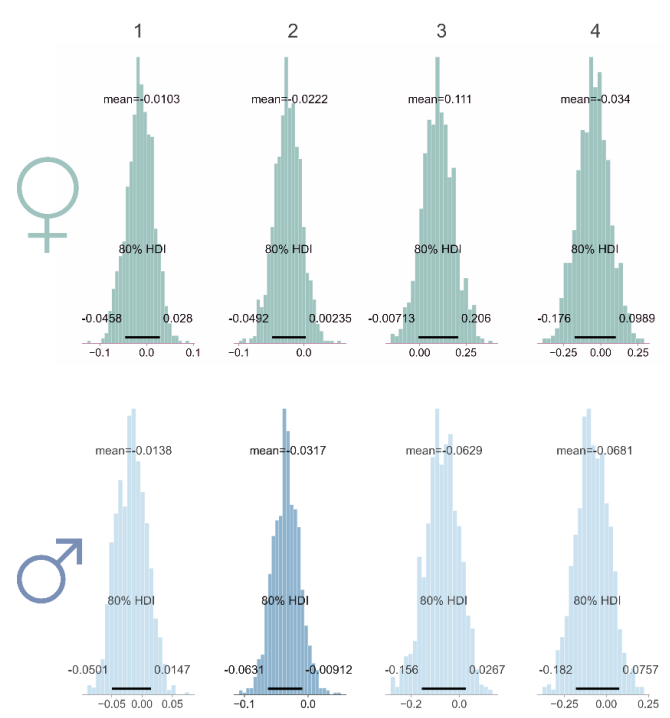
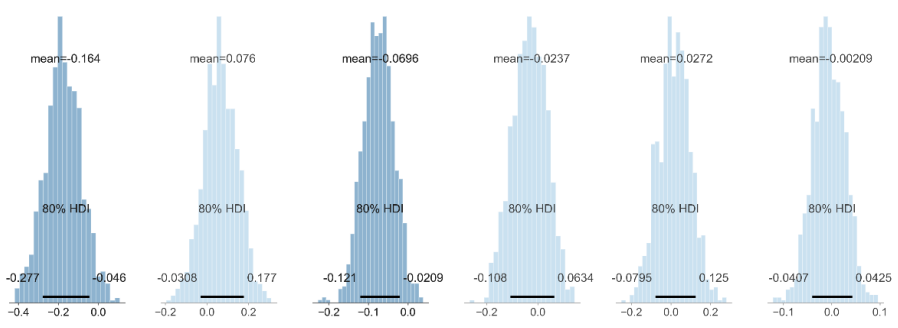

\author{
Hp. Figure 1. K-MMSE Bayesian posteriors
}

\section{Supp. Figure 2. BN Bayesian posteriors}

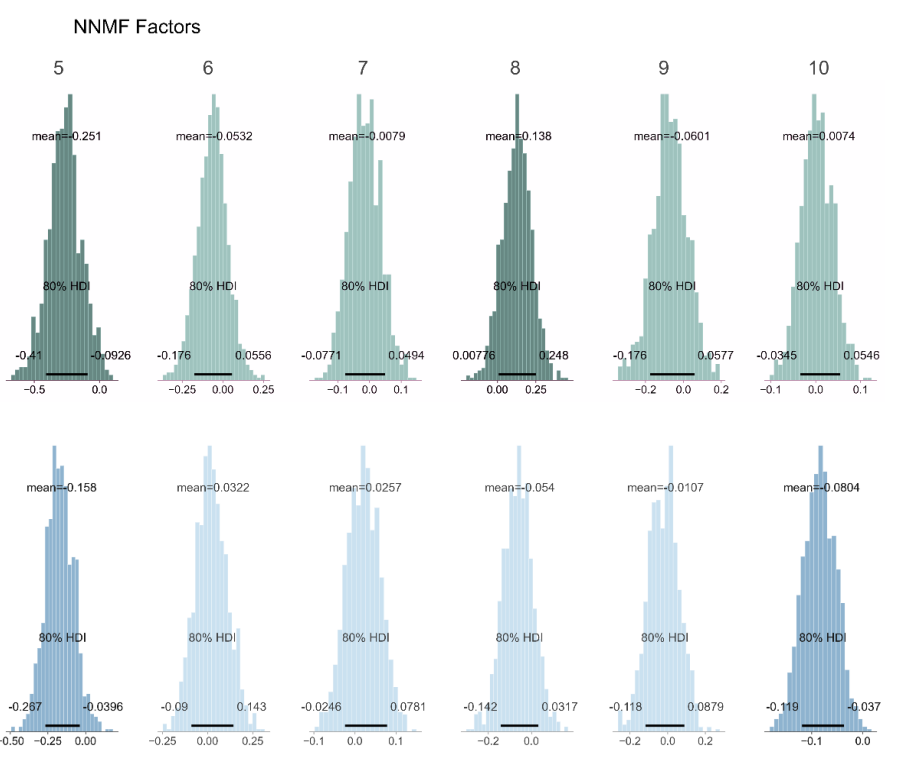


bioRxiv preprint doi: https://doi.org/10.1101/2022.01.18.474989; this version posted January $18,2022$. The copyright holder for this preprint (which was not certified by peer review) is the author/funder, who has granted bioRxiv a license to display the preprint in perpetuity. It is made available under aCC-BY-NC-ND 4.0 International license.

SF
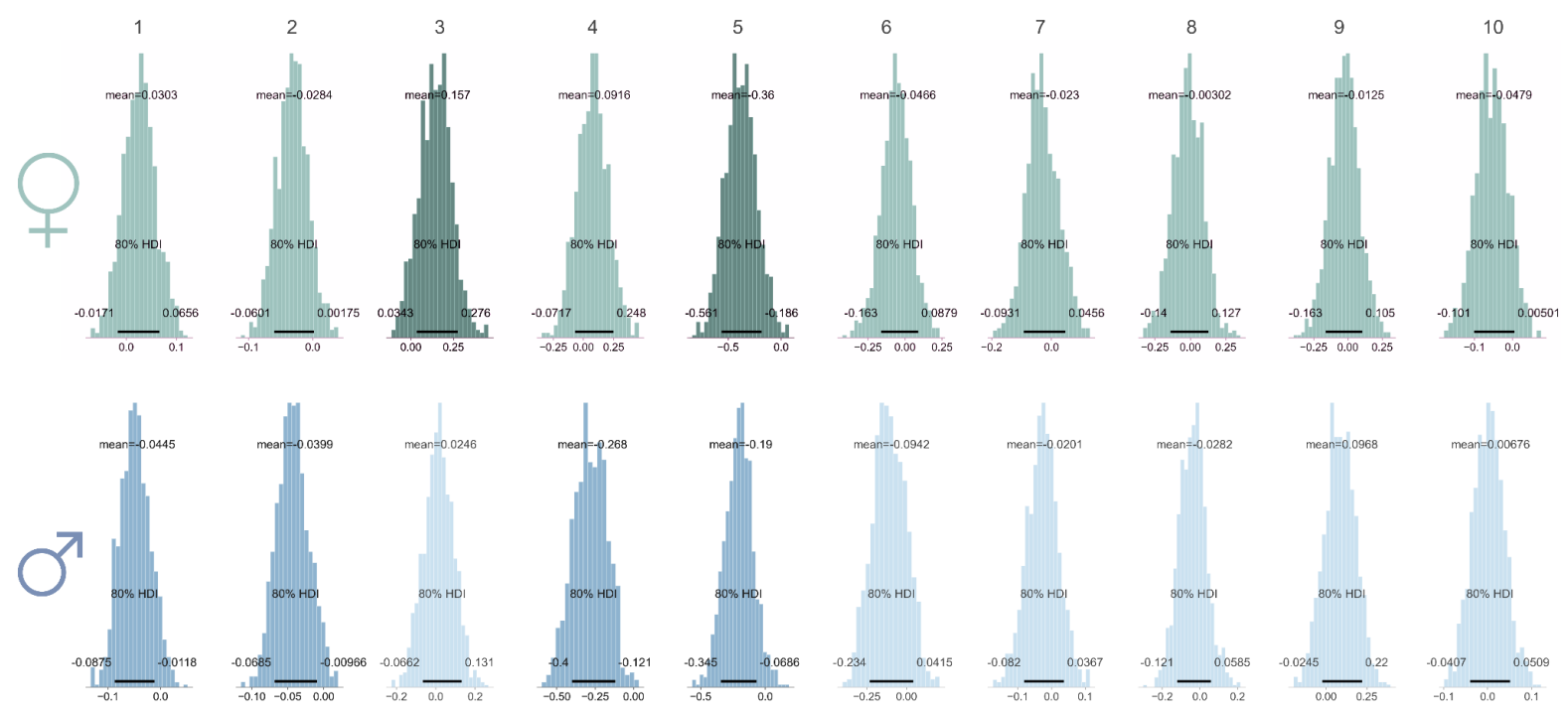

\section{Supp. Figure 3. SF Bayesian posteriors}

PF
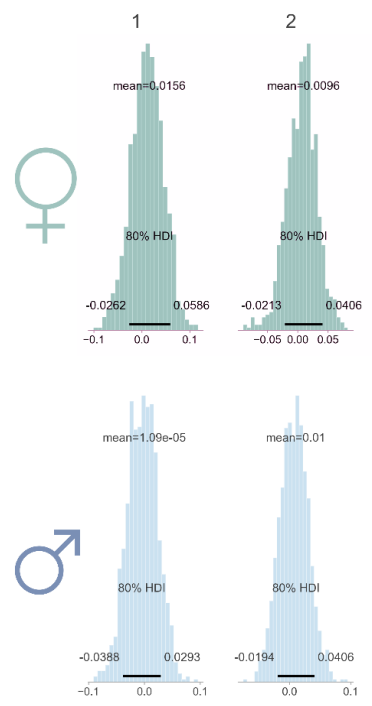
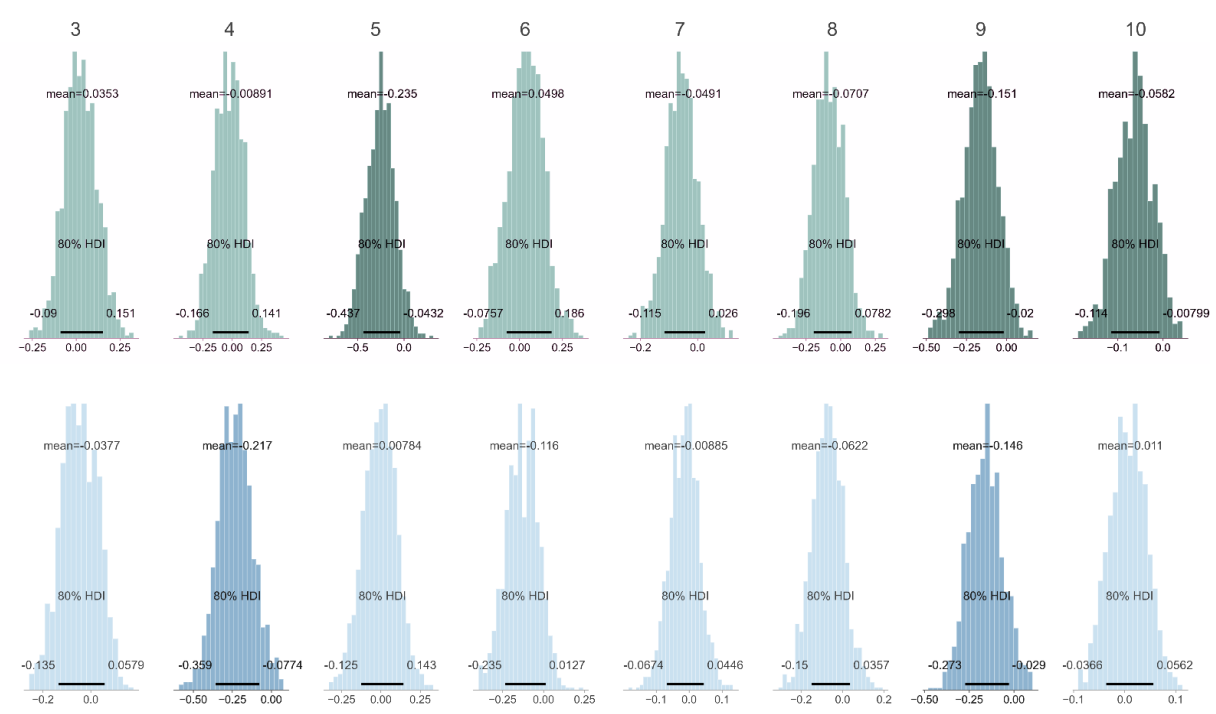

\section{Supp. Figure 4. PF Bayesian posteriors}


SVLT immediate recall
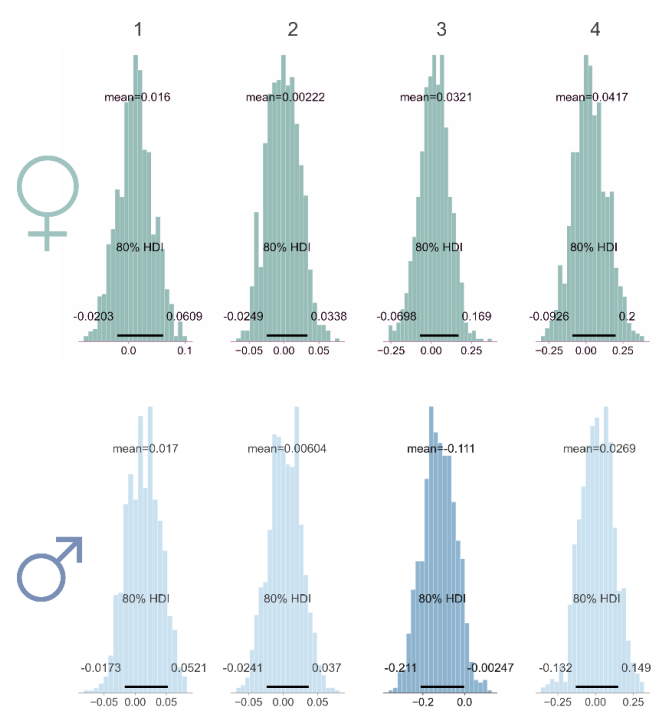
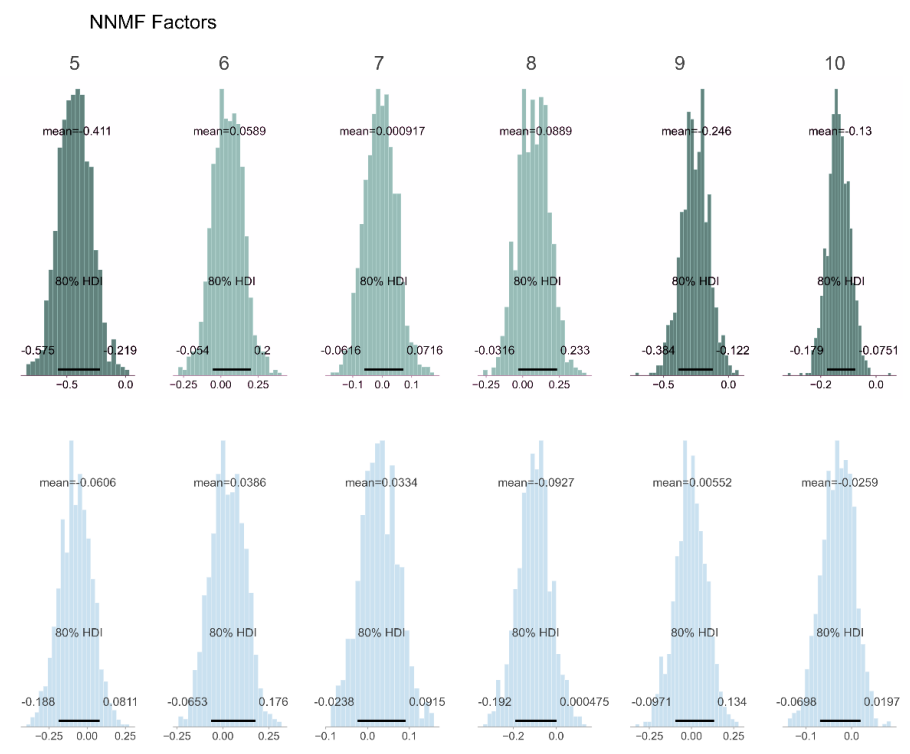

\section{Supp. Figure 5. SVLT immediate recall Bayesian posteriors}

SVLT delayed recall
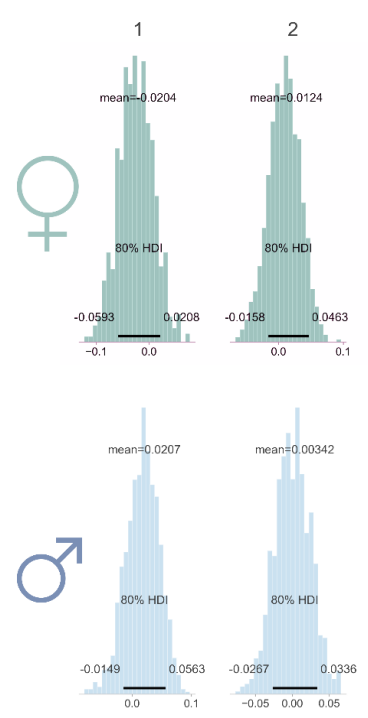
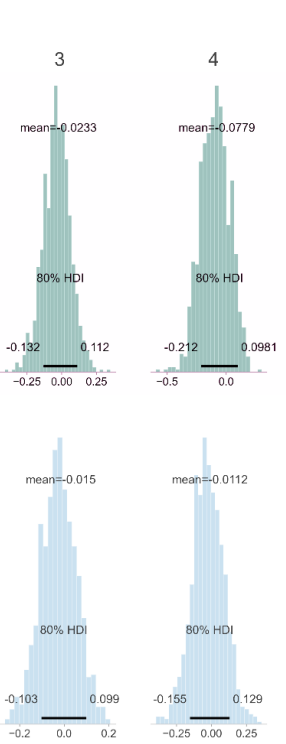

NNMF Factors
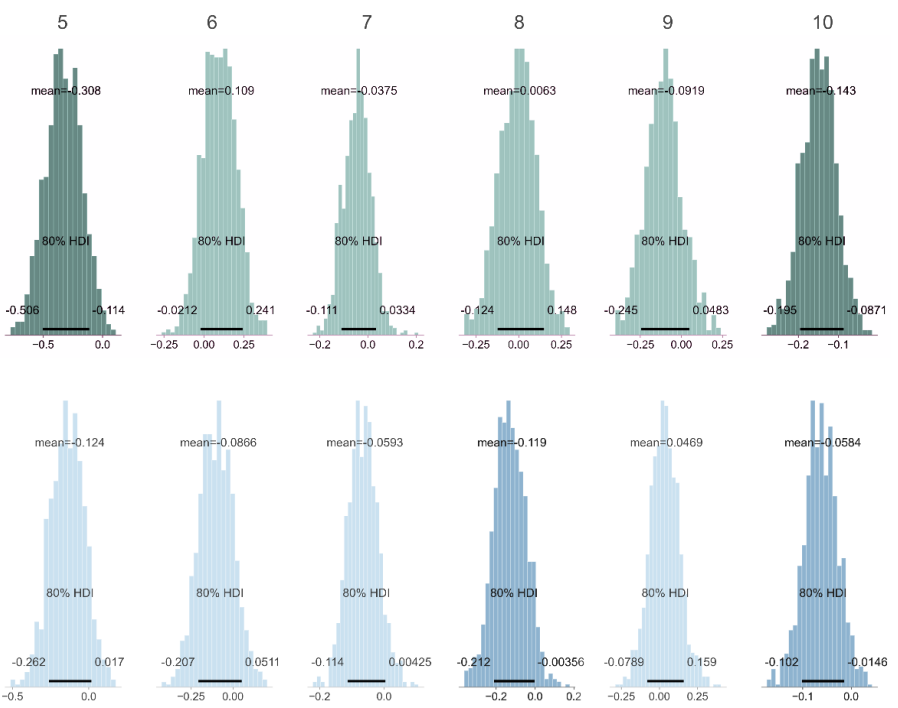

\section{Supp. Figure 6. SVLT delayed recall Bayesian posteriors}


SVLT recognition
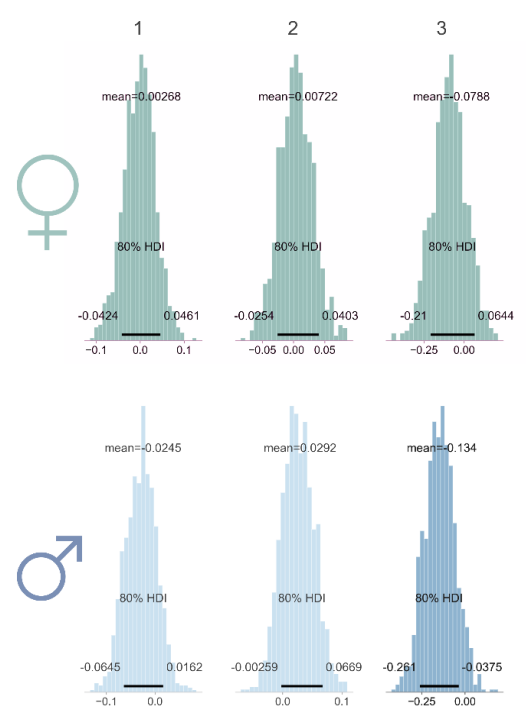
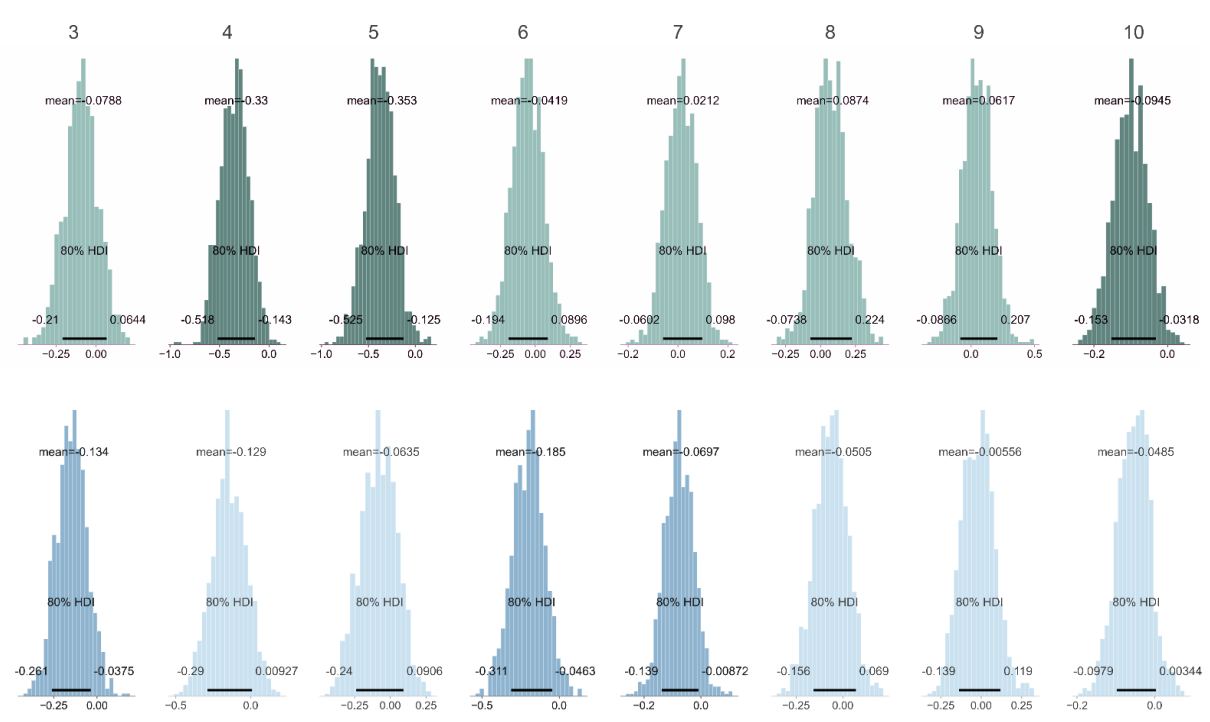

\section{Supp. Figure 7. SVLT recognition Bayesian posteriors}

RCFT delayed recall
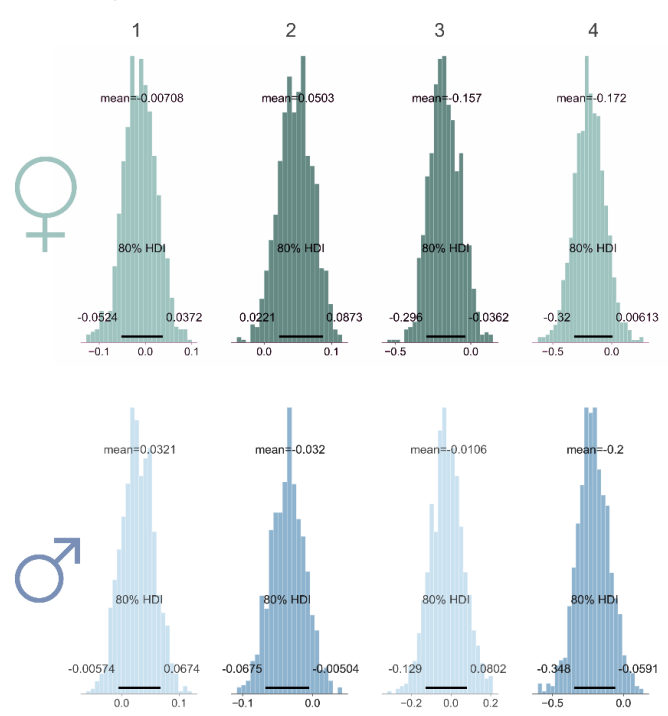
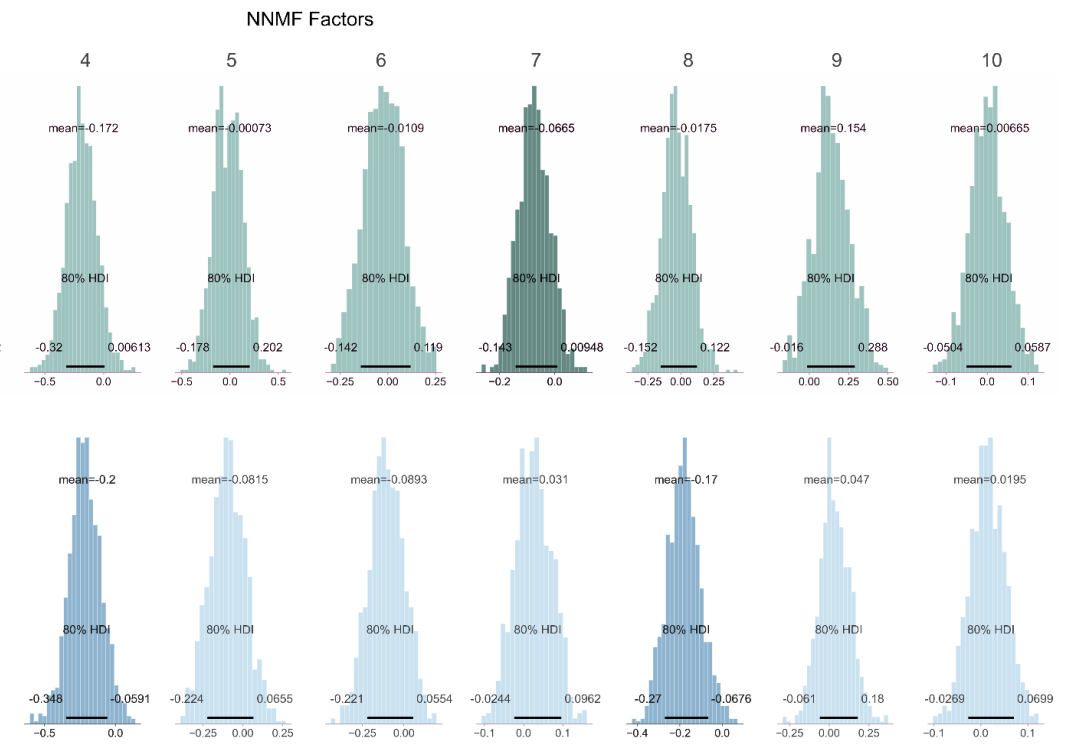

\section{Supp. Figure 8. RCFT delayed recall Bayesian posteriors}


RCFT
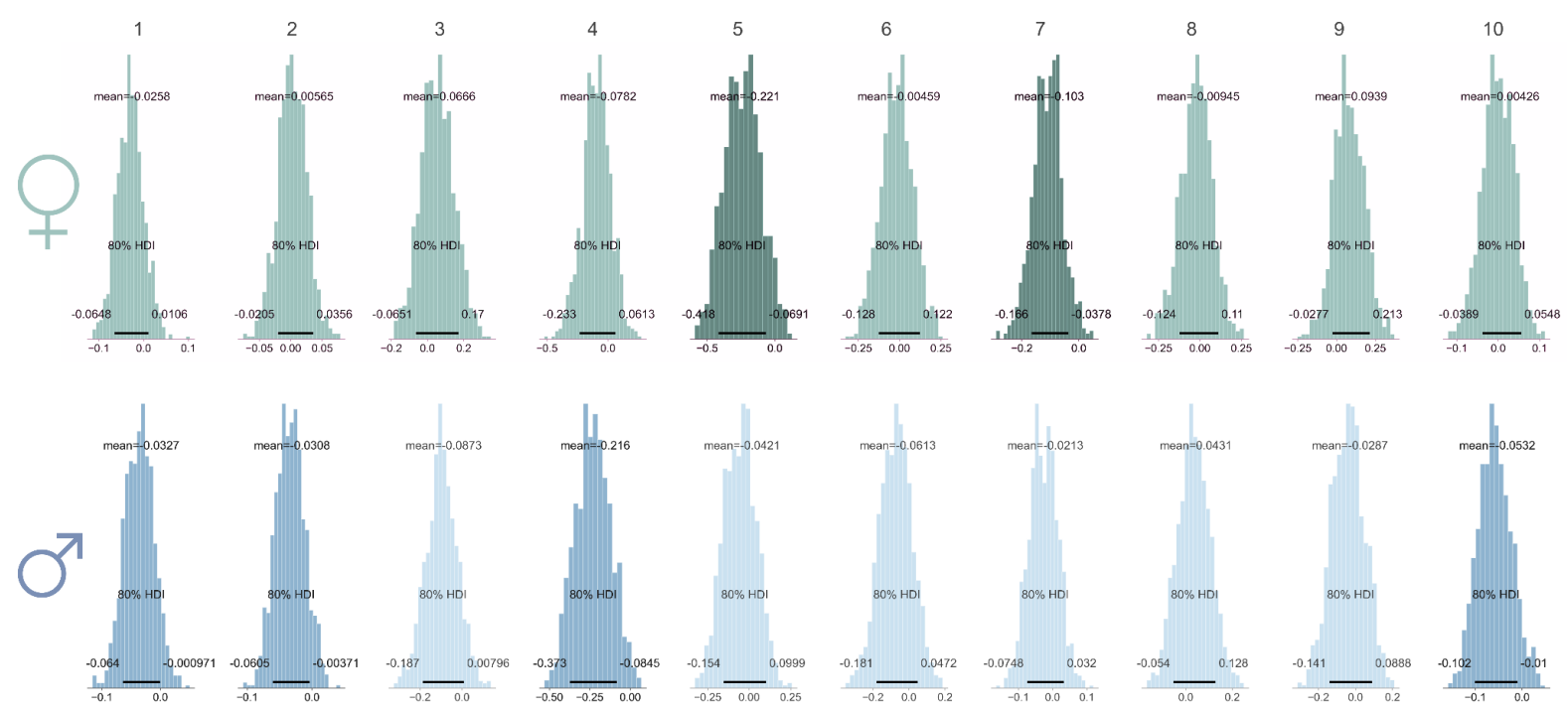

\section{Supp. Figure 9. RCFT Bayesian posteriors}
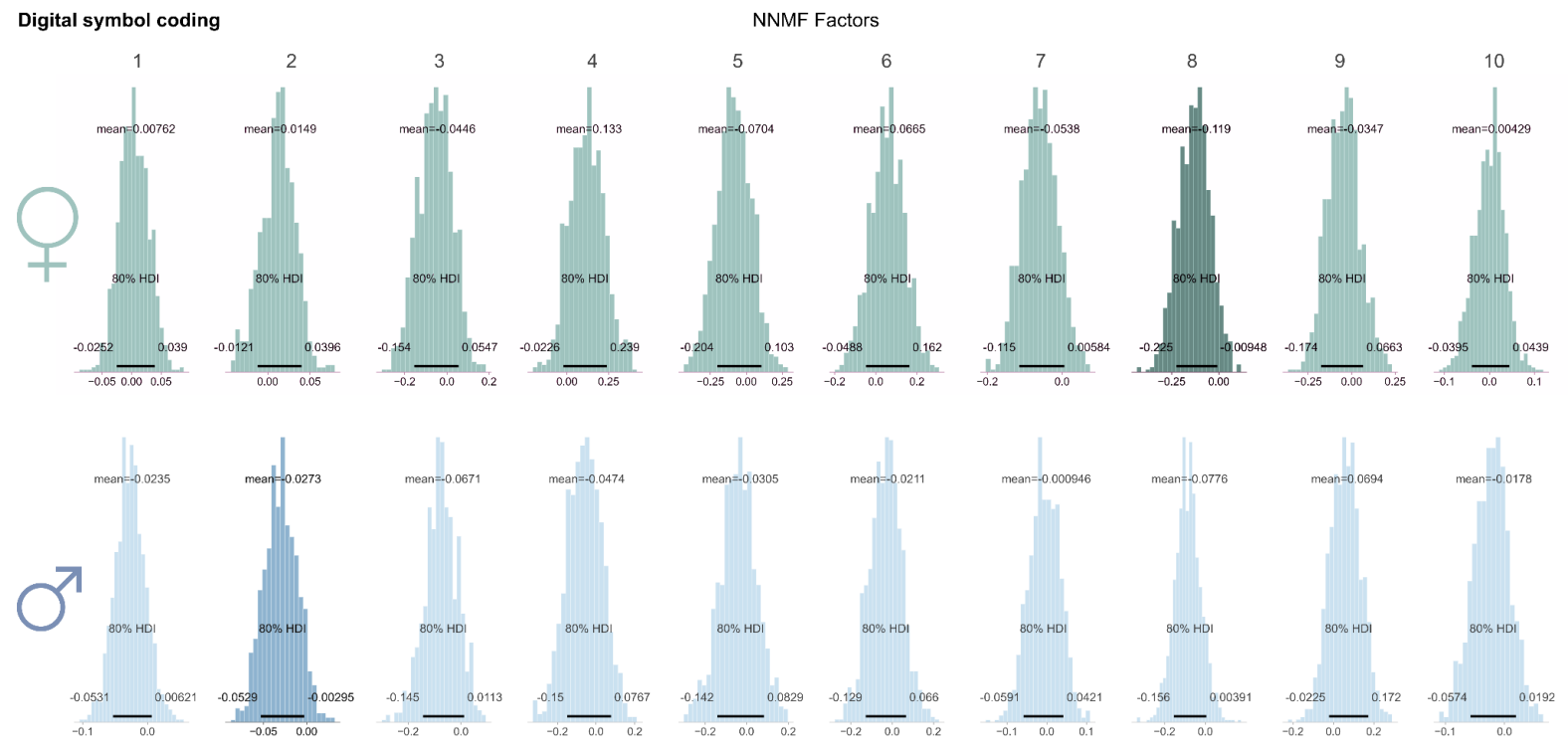

\section{Supp. Figure 10. Digital symbol coding Bayesian posteriors}


TMT-A
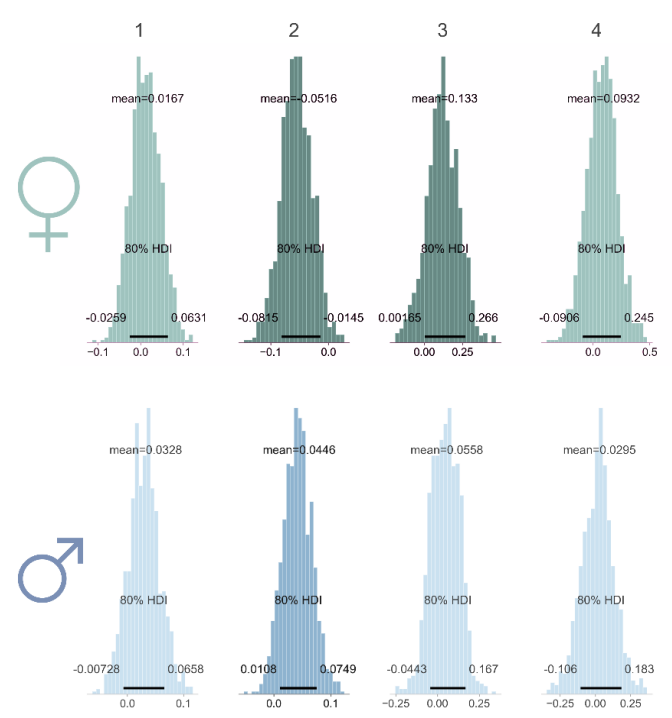

NNMF Factors
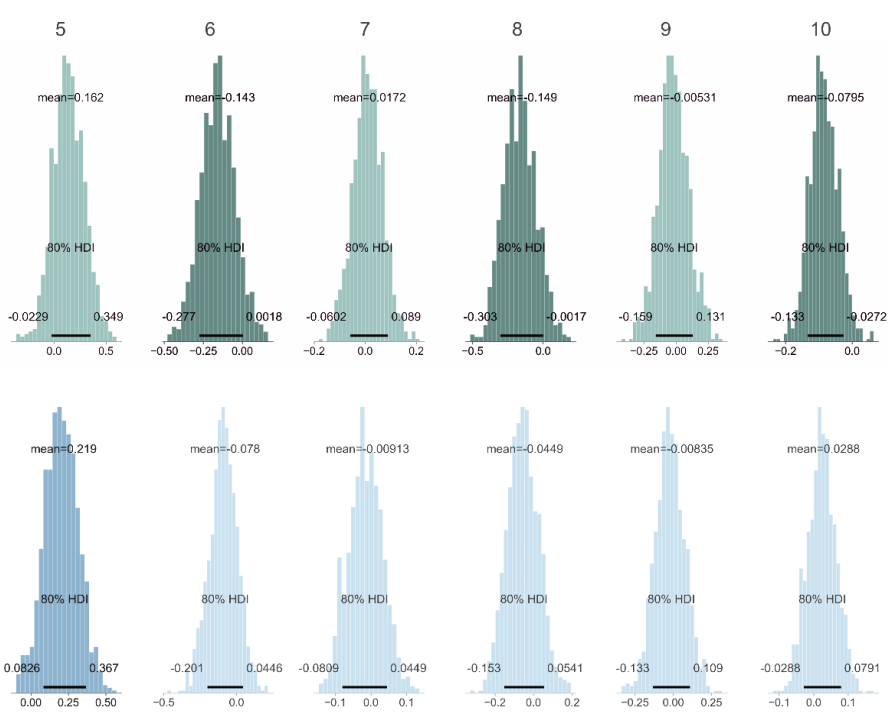

\section{Supp. Figure 11. TMT-A Bayesian posteriors}

TMT-B
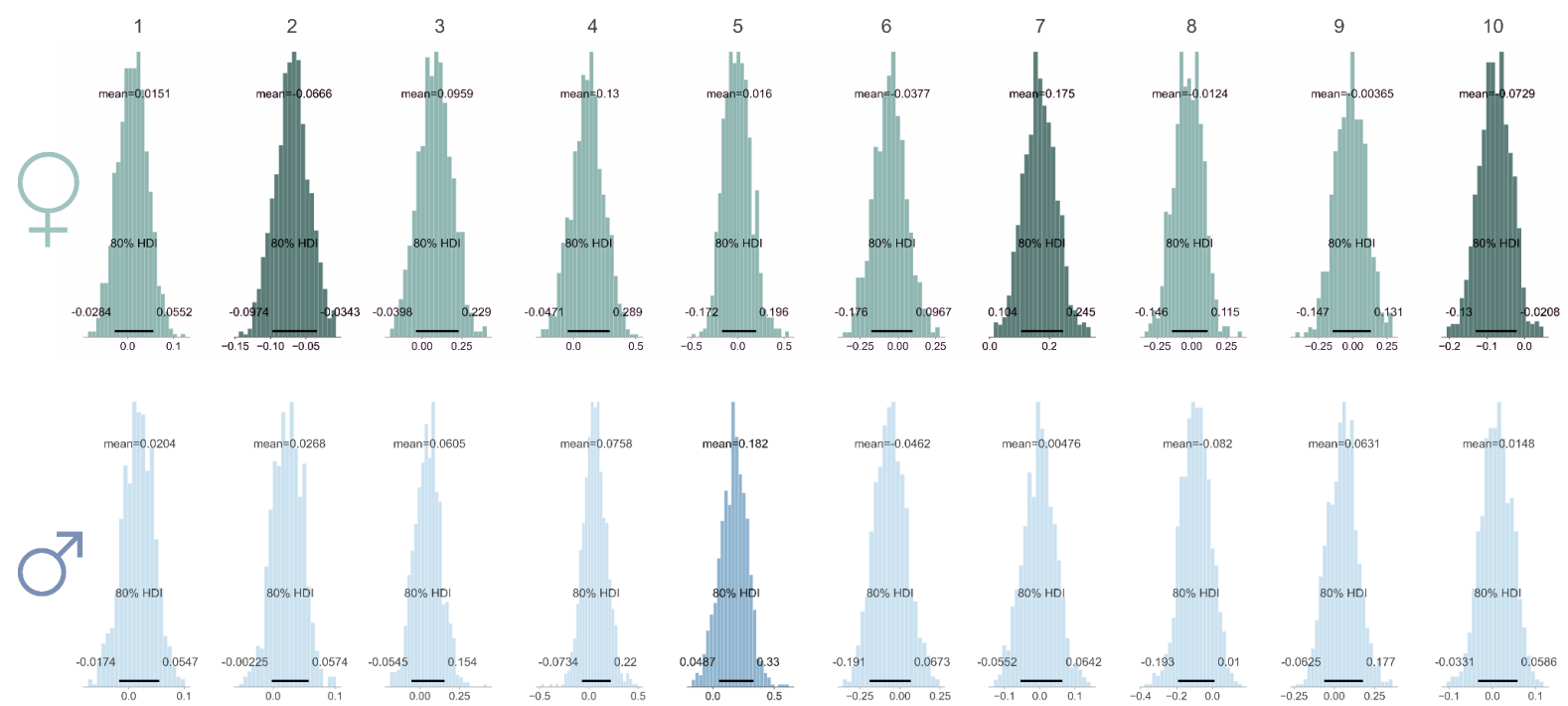

\section{Supp. Figure 12. TMT-B Bayesian posteriors}

